\title{
Diffusive Logistic Equations with Degenerate Boundary Conditions
}

\author{
Kazuaki Taira
}

Dedicated to Professor Mitsuru Ikawa on the occasion of his 60th birthday

\begin{abstract}
The purpose of this paper is to provide a careful and accessible exposition of static bifurcation theory for a class of degenerate boundary value problems for diffusive logistic equations with indefinite weights that model population dynamics in environments with spatial heterogeneity. We discuss the changes that occur in the structure of the positive solutions as a parameter varies near the first eigenvalue of the linearized problem, and prove that the most favorable situations will occur if there is a relatively large favorable region (with good resources and without crowding effects) located some distance away from the boundary of the environment.
\end{abstract}

Mathematics Subject Classification (2000). 35J65, 35P30, 35J25, 92D25.

Keywords. Diffusive logistic equation, degenerate boundary condition, positive solution.

\section{Introduction and Main Results}

Let $D$ be a bounded domain of Euclidean space $\mathbf{R}^{N}, N \geq 3$, with smooth boundary $\partial D$; its closure $\bar{D}=D \cup \partial D$ is an $N$-dimensional, compact smooth manifold with boundary. In this paper we study the following semilinear elliptic boundary value problem:

Here:

$$
\begin{cases}-\Delta u=\lambda(m(x)-h(x) u) u & \text { in } D \\ B u:=a\left(x^{\prime}\right) \frac{\partial u}{\partial \mathbf{n}}+b\left(x^{\prime}\right) u=0 & \text { on } \partial D .\end{cases}
$$

(1) $\Delta=\partial^{2} / \partial x_{1}^{2}+\partial^{2} / \partial x_{2}^{2}+\cdots+\partial^{2} / \partial x_{N}^{2}$ is the usual Laplacian.

(2) $\lambda$ is a real parameter.

(3) $m(x)$ is a real-valued, continuous function on $\bar{D}$. 
(4) $h(x)$ is a real-valued, $C^{1}$ function on $\bar{D}$.

(5) $a\left(x^{\prime}\right)$ and $b\left(x^{\prime}\right)$ are non-negative, smooth functions on $\partial D$.

(6) $\mathbf{n}=\left(n_{1}, n_{2}, \ldots, n_{N}\right)$ is the unit exterior normal to the boundary $\partial D$ (see Figure 1.1).

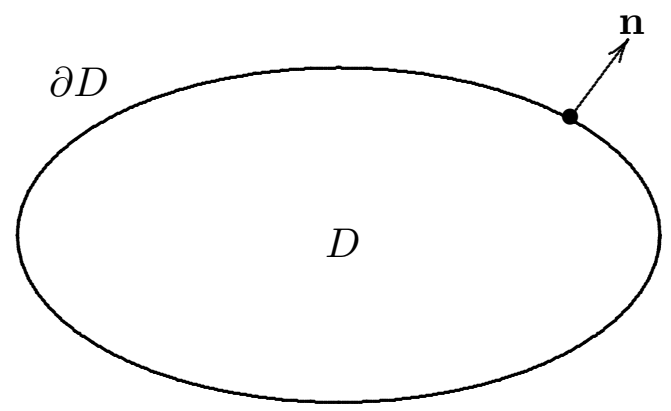

FIGURE 1.1

The main purpose of this work is to study the existence and uniqueness of positive solutions of problem (1.1), substantially improving the previous paper [24]. It should be emphasized that semilinear Dirichlet eigenvalue problems for diffusive logistic equations with discontinuous coefficients are studied in the recent paper [26].

We discuss our motivation and some of the modeling process leading to problem (1.1). The basic interpretation of the various terms in problem (1.1) is that $u(x)$ represents the population density of a species inhabiting the region $D$. The members of the population are assumed to move about $D$ via the type of random walks occurring in Brownian motion that is modeled by the diffusive term $(1 / \lambda) \Delta$; hence $1 / \lambda$ represents the diffusion rate, so small values of $\lambda$ the population spreads more rapidly than for larger values of $\lambda$. The local rate of change in the population density is described by the density dependent term $m(x)-h(x) u$. In this term, $m(x)$ describes the rate at which the population would grow or decline at the location $x$ in the absence of crowding or limitations on the availability of resources. The sign of $m(x)$ will be positive on favorable habitats for population growth and negative on unfavorable ones. Specifically $m(x)$ may be considered as a food source or any resource that will be good in some areas and bad in some others. The term $-h(x) u$ describes the effects of crowding on the growth rate of the population at the location $x$; these effects are assumed to be independent of those determining the growth rate at low densities. The size of $h(x)$ describes the strength of the crowding effects.

On the other hand, in terms of biology, the functions $a\left(x^{\prime}\right)$ and $b\left(x^{\prime}\right)$ measure the hostility of the exterior of the domain. For example, if $a\left(x^{\prime}\right) \equiv 0$ and $b\left(x^{\prime}\right) \equiv 1$ on $\partial D$, then the (Dirichlet) boundary condition $B$ represents that $D$ is surrounded by a completely hostile exterior such that any member of the population which 
reaches the boundary dies immediately; in other words, the exterior of the domain is deadly to the population. If $a\left(x^{\prime}\right) \equiv 1$ and $b\left(x^{\prime}\right) \equiv 0$ on $\partial D$, then the (Neumann) boundary condition $B$ represents that the boundary acts as a barrier, that is, individuals reaching the boundary simply return to the interior. If the exterior is hostile but not completely deadly, then the general boundary condition $B u=$ $a\left(x^{\prime}\right)(\partial u) /(\partial \mathbf{n})+b\left(x^{\prime}\right) u=0$ results.

In this paper we study problem (1.1) under the following two conditions on the functions $m(x), a\left(x^{\prime}\right)$ and $b\left(x^{\prime}\right)$ :

(H.1) The function $m(x)$ takes a positive value in $D$.

(H.2) $a\left(x^{\prime}\right)+b\left(x^{\prime}\right)>0$ on $\partial D$, and $b\left(x^{\prime}\right) \not \equiv 0$ on $\partial D$.

Condition (H.1) implies that there exists a region endowed with a nice food source, while condition (H.2) implies that the exterior of the domain is not totally reflective, that is, the boundary condition $B$ is not the pure Neumann condition. It should be emphasized that problem (1.1) is a degenerate elliptic boundary value problem from an analytical point of view. This is due to the fact that the socalled Shapiro and Lopatinskii complementary condition is violated at the points $x^{\prime} \in \partial D$ where $a\left(x^{\prime}\right)=0$.

First, we study the following linearized boundary value problem:

$$
\begin{cases}A u:=(-\Delta+c(x)) u=g & \text { in } D, \\ B u:=a\left(x^{\prime}\right) \frac{\partial u}{\partial \mathbf{n}}+b\left(x^{\prime}\right) u=\varphi & \text { on } \partial D .\end{cases}
$$

Here $c(x)$ is a real-valued, continuous function on $\bar{D}$. For simplicity, we only consider the case where

$$
c(x) \geq 0 \text { in } D .
$$

We prove an existence and uniqueness theorem for problem (1.2) in the framework of Sobolev spaces of $L^{p}$ style that will play an essential role in the study of problem (1.1).

If $k$ is a positive integer and $1<p<\infty$, we define the Sobolev space

$$
\begin{aligned}
H^{k, p}(D)= & \text { the space of (equivalence classes of) functions } \\
& u \in L^{p}(D) \text { whose derivatives } D^{\alpha} u,|\alpha| \leq k, \text { in the } \\
& \text { sense of distributions are in } L^{p}(D),
\end{aligned}
$$

and the boundary space

$$
\begin{gathered}
B^{k-1 / p, p}(\partial D)=\text { the space of the boundary values }\left.u\right|_{\partial D} \text { of functions } \\
\qquad u \in H^{k, p}(D) .
\end{gathered}
$$

In the space $B^{k-1 / p, p}(\partial D)$, we define a norm

$$
|\varphi|_{B^{k-1 / p, p}(\partial D)}=\inf \left\{\|u\|_{H^{k, p}(D)}: u \in H^{k, p}(D),\left.u\right|_{\partial D}=\varphi\right\} .
$$

The space $B^{k-1 / p, p}(\partial D)$ is a Banach space with respect to the norm $|\cdot|_{B^{k-1 / p, p}(\partial D)}$; more precisely, it is a Besov space (cf. [2], [28]). 
We introduce a subspace of $B^{1-1 / p, p}(\partial D)$ which is associated with the boundary condition

$$
B u=a\left(x^{\prime}\right) \frac{\partial u}{\partial \mathbf{n}}+b\left(x^{\prime}\right) u
$$

in the following way: We let

$$
\begin{aligned}
& B_{*}^{1-1 / p, p}(\partial D) \\
= & \left\{\varphi=a\left(x^{\prime}\right) \varphi_{1}+b\left(x^{\prime}\right) \varphi_{2}: \varphi_{1} \in B^{1-1 / p, p}(\partial D), \varphi_{2} \in B^{2-1 / p, p}(\partial D)\right\},
\end{aligned}
$$

and define a norm

$$
\begin{aligned}
& |\varphi|_{B_{*}^{1-1 / p, p}(\partial D)} \\
= & \inf \left\{\left|\varphi_{1}\right|_{B^{1-1 / p, p}(\partial D)}+\left|\varphi_{2}\right|_{B^{2-1 / p, p}(\partial D)}: \varphi=a\left(x^{\prime}\right) \varphi_{1}+b\left(x^{\prime}\right) \varphi_{2}\right\} .
\end{aligned}
$$

It is easy to verify that the space $B_{*}^{1-1 / p, p}(\partial D)$ is a Banach space with respect to the norm $|\cdot|_{B_{*}^{1-1 / p, p}(\partial D)}$.

We remark that the space $B_{*}^{1-1 / p, p}(\partial D)$ is an "interpolation space" between the Besov spaces $B^{2-1 / p, p}(\partial D)$ and $B^{1-1 / p, p}(\partial D)$. In fact, we have

$$
\begin{cases}B_{*}^{1-1 / p, p}(\partial D)=B^{2-1 / p, p}(\partial D) & \text { if } a\left(x^{\prime}\right) \equiv 0 \text { on } \partial D \\ B_{*}^{1-1 / p, p}(\partial D)=B^{1-1 / p, p}(\partial D) & \text { if } a\left(x^{\prime}\right)>0 \text { on } \partial D .\end{cases}
$$

Our first main result of this paper is stated as follows:

Theorem 1.1. Assume that conditions (H.2) and (1.3) are satisfied. Then the mapping

$$
\mathcal{A}:=(A, B): H^{2, p}(D) \longrightarrow L^{p}(D) \bigoplus B_{*}^{1-1 / p, p}(\partial D)
$$

is an algebraic and topological isomorphism for all $N<p<\infty$.

Now, in order to study problem (1.1) we consider the following linearized eigenvalue problem:

$$
\begin{cases}-\Delta \varphi=\lambda m(x) \varphi & \text { in } D \\ B \varphi=0 & \text { on } \partial D .\end{cases}
$$

The next theorem asserts that the first eigenvalue of problem (1.4) is algebraically simple and its corresponding eigenfunction is positive, which is a generalization of a result due to Manes and Micheletti [17] (see [7, Theorem 1.13]) to the degenerate case:

Theorem 1.2. Assume that conditions (H.1) and (H.2) are satisfied. Then the first eigenvalue $\lambda_{1}(m)$ of problem (1.4) is positive and algebraically simple, and its corresponding eigenfunction $\psi_{1}(x) \in H^{2, p}(D), N<p<\infty$, may be chosen to be positive everywhere in D. Moreover, no other eigenvalues have positive eigenfunctions. 
Here it should be noticed that we have, by Sobolev's imbedding theorem,

$$
H^{2, p}(D) \subset C^{1}(\bar{D})
$$

since $2-N / p>1$ for $N<p<\infty$.

By the Rayleigh principle, we can prove that the first eigenvalue $\lambda_{1}(m)$ is characterized by the variational formula

$$
\lambda_{1}(m)=\inf \left\{\frac{(-\Delta \phi, \phi) L^{2}(D)}{\int_{D} m(x) \phi^{2} d x}: \phi \in H^{2,2}(D), B \phi=0, \int_{D} m(x) \phi^{2} d x>0\right\} .
$$

A biological interpretation of Theorem 1.2 is that if there is a favorable region, then the models we consider predict persistence for a population, since the existence of the first positive eigenvalue is equivalent to the existence of a positive density function describing the distribution of the population of $D$. It is worthwhile to point out here that the first eigenvalue $\lambda_{1}(m)$ will tend to be smaller in situations where favorable and unfavorable habitats are closely intermingled (producing cancellation effects), and larger when the favorable region consists of a relatively small number of relatively large isolated components.

A solution $u(x) \in C^{2}(\bar{D})$ of problem (1.1) is said to be non-trivial if it does not identically equal zero on $\bar{D}$. We call a non-trivial solution $u$ of problem (1.1) a positive solution if $u(x) \geq 0$ on $\bar{D}$.

In this paper we discuss the changes that occur in the structure of the positive solutions as the parameter $\lambda$ varies near the first eigenvalue $\lambda_{1}(m)$ under the condition that

(H.3) The function $m(x)$ attains both positive and negative values in $D$.

Assume that $h(x)$ is a function in $C^{1}(\bar{D})$ such that

$$
h(x) \geq 0 \text { on } \bar{D},
$$

and let

$$
D_{0}(h)=\text { the interior of the set }\{x \in D: h(x)=0\} .
$$

In this paper we study the case where $h(x)>0$ on the boundary $\partial D$. More precisely, our structural condition on the function $h(x)$ is stated as follows (see Figure 1.2):

(Z) The open set $D_{0}(h)$ consists of a finite number of connected components with smooth boundary, say $D_{0}^{i}(h), 1 \leq i \leq \ell$, which are bounded away from the boundary $\partial D$.

In the Dirichlet case, condition $(\mathrm{Z})$ can be weakened such that the function $h(x)$ may vanish on the boundary $\partial D$ (see [9, Theorem 3.5]).

We consider the Dirichlet eigenvalue problem in each connected component $D_{0}^{i}(h), 1 \leq i \leq \ell$,

$$
\begin{cases}-\Delta \varphi=\lambda m(x) \varphi & \text { in } D_{0}^{i}(h), \\ \varphi=0 & \text { on } \partial D_{0}^{i}(h),\end{cases}
$$

and let

$$
\lambda_{1}\left(D_{0}^{i}(h)\right)=\text { the first eigenvalue of problem (1.6). }
$$




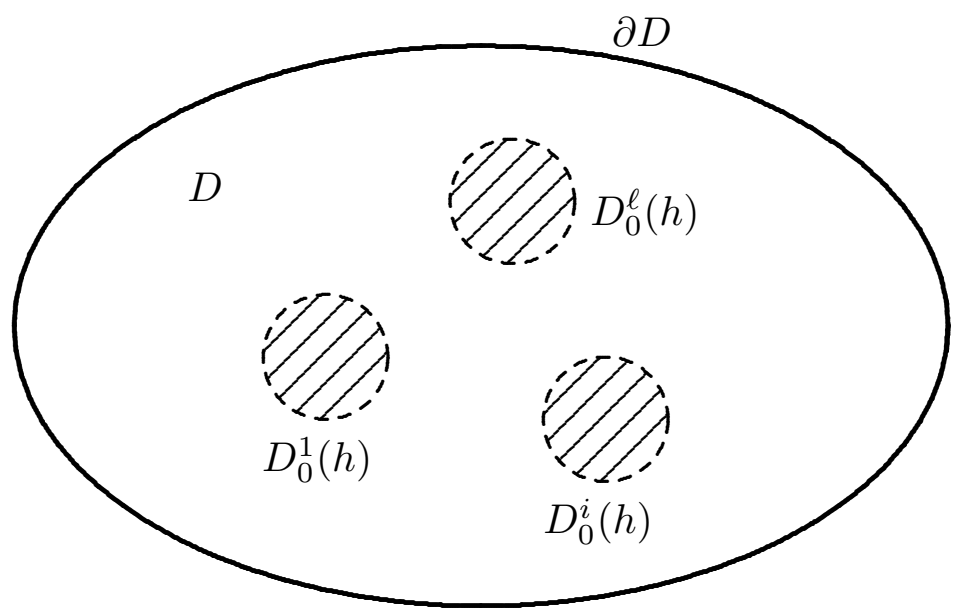

FIGURE 1.2

By the Rayleigh principle, we know that the first eigenvalue $\lambda_{1}\left(D_{0}^{i}(h)\right)$ is given by the variational formula

$$
\begin{aligned}
& \lambda_{1}\left(D_{0}^{i}(h)\right) \\
& =\inf \left\{\frac{\int_{D_{0}^{i}(h)}|\nabla \psi|^{2} d x}{\int_{D_{0}^{i}(h)} m(x) \psi^{2} d x}: \psi \in H_{0}^{1}\left(D_{0}^{i}(h)\right), \int_{D_{0}^{i}(h)} m(x) \psi^{2} d x>0\right\} .
\end{aligned}
$$

Here $H_{0}^{1}\left(D_{0}^{i}(h)\right)$ is the closure of smooth functions with compact support in $D_{0}^{i}(h)$ in the Sobolev space $H^{1,2}\left(D_{0}^{i}(h)\right)$.

We let

$$
\mu_{1}\left(D_{0}(h)\right)=\min \left\{\lambda_{1}\left(D_{0}^{1}(h)\right), \lambda_{1}\left(D_{0}^{2}(h)\right), \ldots, \lambda_{1}\left(D_{0}^{\ell}(h)\right)\right\} .
$$

Now we can state our main result that is a generalization of Fraile et al. [9, Theorem 3.5] to the degenerate case (cf. [21, Theorem 3.2], [24, Theorem 1.2]):

Theorem 1.3. In addition to condition (H.2), assume that the function $m(x) \in$ $C^{\theta}(\bar{D}), 0<\theta<1$, satisfies condition (H.3) and further that the function $h(x) \in$ $C^{1}(\bar{D})$ satisfies condition $(\mathrm{Z})$ and that each set $\left\{x \in D_{0}^{i}(h): m(x)>0\right\}, 1 \leq$ $i \leq \ell$, has positive measure. Then problem (1.1) has a unique positive solution $u(\lambda) \in C^{2+\theta}(\bar{D})$ for every $\lambda \in\left(\lambda_{1}(m), \mu_{1}\left(D_{0}(h)\right)\right)$. For any $\lambda \geq \mu_{1}\left(D_{0}(h)\right)$, there exists no positive solution of problem (1.1). Moreover, we have

$$
\lim _{\lambda \rightarrow \mu_{1}\left(D_{0}(h)\right)}\|u(\lambda)\|_{L^{2}(D)}=+\infty
$$

and also

$$
\lim _{\lambda \rightarrow \lambda_{1}(m)}\|u(\lambda)\|_{C^{2+\theta}(\bar{D})}=0
$$


Our situation may be represented schematically by the following bifurcation diagram, Figure 1.3:

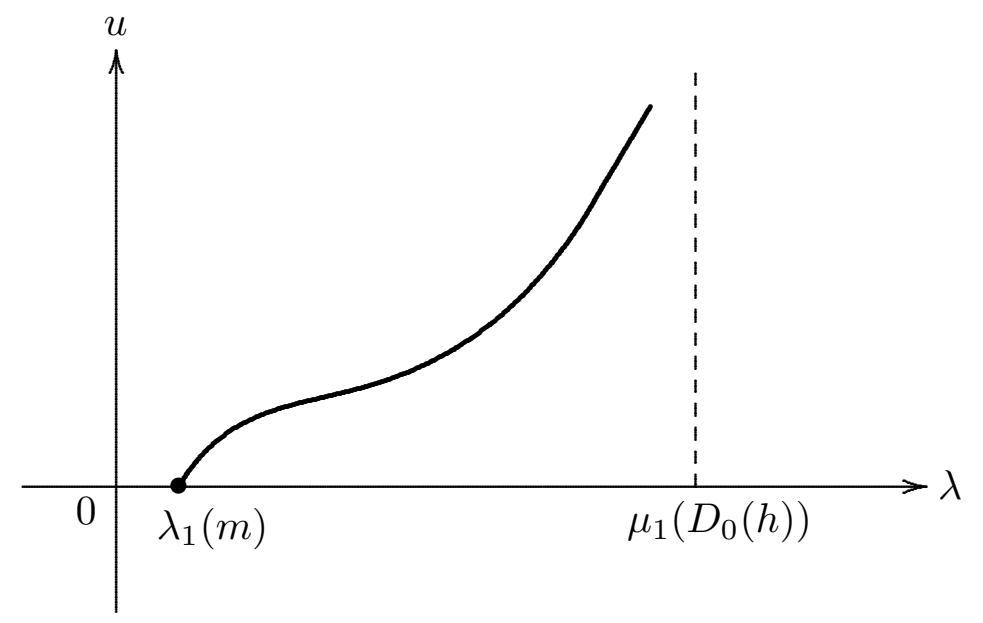

FIGURE 1.3

Remark 1.1. Theorem 1.3 may be proved by using the super-sub-solution method, just as in the proof of Fraile et al. [9, Theorems 3.5 and 4.6], if assertion (1.7) is replaced by a weaker one

$$
\lim _{\lambda \rightarrow \mu_{1}\left(D_{0}(h)\right)}\|u(\lambda)\|_{C(\bar{D})}=+\infty .
$$

Theorem 1.3 asserts that assertion (1.7) holds true if $N \geq 3$. It should be emphasized that an estimate of the growth rate of the total size $\|u(\lambda)\|_{L^{1}(D)}=\int_{D} u(\lambda) d x$ of the positive steady states $u(\lambda)$ as $\lambda \uparrow \mu_{1}\left(D_{0}(h)\right)$ is of crucial importance from the viewpoint of population dynamics as in estimate (8.1) in Section 8.

Rephrased, Theorem 1.3 asserts that the models we consider predict persistence for a population if its diffusion rate $1 / \lambda$ is below the critical value $1 / \lambda_{1}(m)$ depending on the coefficient $m(x)$ which describes the growth rate and if it is above the critical value $1 / \mu_{1}\left(D_{0}(h)\right)$ depending on the coefficient $h(x)$ which describes the strength of the crowding effects. Theorem 1.3 also asserts that, in a certain sense, the most favorable situations will occur if there is a relatively large favorable region (with good resources and without crowding effects) located some distance away from the boundary of $D$.

The rest of this paper is organized as follows. In Section 2 we summarize the basic definitions and results about ordered Banach spaces and the well-known Kreŭn and Rutman theorem for strongly positive, compact linear operators (Theorem 2.1) that enter naturally in connection with elliptic eigenvalue problems. In Section 3 we study the non-homogeneous boundary value problem (1.2), and we 
prove Theorem 1.1. Our proof is carried out just as in the proof of Brown and Lin [4, Theorem 3.5] by using Theorem 3.10. Section 4 is devoted to the proof of Theorem 1.2. In Section 5 we study the inequalities among the first eigenvalues $\mu_{D}(\lambda), \mu_{N}(\lambda)$ and $\mu(\lambda)$ subject to Dirichlet, Neumann and general boundary conditions, respectively (Theorem 5.3). Sections 6 and 7 are devoted to the proof of Theorem 1.3. Our approach to problem (1.1) is a modification of that of Ouyang [18] adapted to the present context. First, by using Green's formula we prove that if there exists a positive solution $u(\lambda)$ of problem (1.1), then it follows that $\lambda>\lambda_{1}(m)$ (Lemma 6.1). The existence of positive solutions of problem (1.1) near the point $\left(\lambda_{1}(m), 0\right)$ follows by applying local static bifurcation theory from a simple eigenvalue due to Crandall and Rabinowitz [6] (Theorem 6.2). Next, by making use of the implicit function theorem we prove that there exists a critical value $\bar{\lambda}(h) \in\left(\lambda_{1}(m), \mu_{1}\left(D_{0}(h)\right)\right]$ such that problem (1.1) has a positive solution $u(\lambda)$ for all $\lambda \in\left(\lambda_{1}(m), \bar{\lambda}(h)\right)$ (Lemma 6.4). The formula $\bar{\lambda}(h)=\mu_{1}\left(D_{0}(h)\right)$ follows from the uniqueness of a bifurcation point and the comparison principle (Theorem 7.3). In the final Section 8 we consider problem (1.1) under the condition that $h(x)>0$ on $\bar{D}$, and prove an estimate of the growth rate of the total size $\|u(\lambda)\|_{L^{1}(D)}$ of the positive steady states $u(\lambda)$ (Theorem 8.1).

The author is grateful to Kenichiro Umezu for fruitful conversations while working on this paper. This research is partially supported by Grant-in-Aid for General Scientific Research (No. 16340031), Ministry of Education, Culture, Sports, Science and Technology, Japan.

\section{Theory of Ordered Banach Spaces}

A general class of semilinear second-order elliptic boundary value problems satisfies the maximum principle. Roughly speaking, this additional information means that the operators associated with the boundary value problems are compatible with the natural ordering of the underlying function spaces. Consequently, we are led to the study of nonlinear equations in the framework of ordered Banach spaces.

\subsection{Ordered Banach Spaces and the Kreĭn and Rutman Theorem}

Let $X$ be a non-empty set. An ordering $\leq$ in $X$ is a relation in $X$ which is reflexive, transitive and antisymmetric. A non-empty set together with an ordering is called an ordered set.

Let $V$ be a real vector space. An ordering $\leq$ in $V$ is said to be linear if the following two conditions are satisfied:

(i) If $x, y \in V$ and $x \leq y$, then we have $x+z \leq y+z$ for all $z \in V$.

(ii) If $x, y \in V$ and $x \leq y$, then we have $\alpha x \leq \alpha y$ for all $\alpha \geq 0$.

A real vector space together with a linear ordering is called an ordered vector space.

If we let

$$
Q=\{x \in V: x \geq 0\},
$$

then it is easy to verify that the set $Q$ has the following two conditions: 
(iii) If $x, y \in Q$, then $\alpha x+\beta y \in Q$ for all $\alpha, \beta \geq 0$.

(iv) If $x \neq 0$, then at least one of $x$ and $-x$ does not belong to $Q$, that is, $Q \cap(-Q)=\{0\}$.

The set $Q$ is called the positive cone of the ordering $\leq$.

Let $E$ be a Banach space $E$ with a linear ordering $\leq$. The Banach space $E$ is called an ordered Banach space if the positive cone $P$ is closed in $E$. For $x, y \in E$, we write

$$
\begin{array}{ll}
x \geq y & \text { if } x-y \in P, \\
x>y & \text { if } x-y \in P \backslash\{0\} .
\end{array}
$$

If the interior $\operatorname{Int}(P)$ is non-empty, then we write

$$
x \gg y \text { if } x-y \in \operatorname{Int}(P) .
$$

A linear operator $K: E \rightarrow E$ is said to be strongly positive if $K x$ belongs to $\operatorname{Int}(P)$ for every $x \in P \backslash\{0\}$ :

$$
x>0 \Longrightarrow K x \gg 0 .
$$

Then the well-known Krel̆n and Rutman theorem for strongly positive, compact linear operators reads as follows (see [15, Theorem 6.3]):

Theorem 2.1. Let $(E, P)$ be an ordered Banach space with non-empty $\operatorname{Int}(P)$ and $K: E \rightarrow E$ a linear operator. If $K$ is strongly positive and compact, then we have the following three assertions:

(1) $r:=\lim _{n \rightarrow \infty} \sqrt[n]{\left\|K^{n}\right\|}>0$ and $r$ is the unique eigenvalue of $K$ having $a$ positive eigenfunction $x$.

(2) The eigenvalue $r$ is algebraically simple and $x \gg 0$.

(3) The eigenvalue $r$ is greater than all the remaining eigenvalues $\lambda$ of $K: r>|\lambda|$.

The eigenvalue $r$ is called the principal eigenvalue of $K$.

\subsection{Application of the Kreı̆n and Rutman Theorem}

As an application of the Kreı̆n and Rutman theorem, we consider the following non-homogeneous equation: For a given $h>0$ in $E$, find an element $u \in E$ such that

$$
\lambda u-K u=h,
$$

where $\lambda$ is a real parameter.

The next theorem will play an important role in the proof of Theorem 1.3 in the sequel (see [1], [12]):

Theorem 2.2. Let $K: E \rightarrow E$ be a strongly positive, compact linear operator and $r$ its principal eigenvalue. Then we have the following three assertions:

(i) If $\lambda>r$, then equation (2.1) has a unique positive solution $u$ and $u \gg 0$.

(ii) If $\lambda<r$, then equation (2.1) has no positive solution.

(iii) If $\lambda=r$, then equation (2.1) has no solution. 


\section{Elliptic Boundary Value Problems}

In this section we study the following non-homogeneous boundary value problem:

$$
\begin{cases}A u:=(-\Delta+c(x)) u=g & \text { in } D, \\ B u:=a\left(x^{\prime}\right) \frac{\partial u}{\partial \mathbf{n}}+b\left(x^{\prime}\right) u=\varphi & \text { on } \partial D .\end{cases}
$$

Here we recall that:

(1) $\Delta=\partial^{2} / \partial x_{1}^{2}+\partial^{2} / \partial x_{2}^{2}+\cdots+\partial^{2} / \partial x_{N}^{2}$.

(2) $c(x) \in C(\bar{D})$.

(3) $a\left(x^{\prime}\right), b\left(x^{\prime}\right) \in C^{\infty}(\partial D)$.

(4) $\mathbf{n}=\left(n_{1}, n_{2}, \ldots, n_{N}\right)$ is the unit exterior normal to the boundary $\partial D$.

Moreover, we assume that

(H.2) $a\left(x^{\prime}\right)+b\left(x^{\prime}\right)>0$ on $\partial D$, and $b\left(x^{\prime}\right) \not \equiv 0$ on $\partial D$,

and further that the function $c(x)$ satisfies the condition

$$
c(x) \geq 0 \quad \text { in } D \text {. }
$$

\subsection{Proof of Theorem 1.1}

This subsection is devoted to the proof of Theorem 1.1. In fact, we prove the following:

Theorem 3.1. Assume that conditions (H.2) and (1.3) are satisfied. Then the mapping

$$
\mathcal{A}:=(A, B): H^{2, p}(D) \longrightarrow L^{p}(D) \bigoplus B_{*}^{1-1 / p, p}(\partial D)
$$

is an algebraic and topological isomorphism for all $N<p<\infty$. In particular, for any $f \in L^{p}(D)$ and any $\varphi \in B_{*}^{1-1 / p, p}(\partial D)$, there exists a unique solution $u \in H^{2, p}(D)$ of problem (1.2).

Proof. To prove Theorem 3.1, it suffices to show that the operator $\mathcal{A}$ is bijective. Indeed, since the inverse $\mathcal{A}^{-1}$ is a closed operator, we obtain that $\mathcal{A}^{-1}$ is continuous if we apply Banach's closed graph theorem (see [30, Chapter II, Section 6, Theorem 1]).

The proof of Theorem 3.1 is divided into four steps.

Step 1: First, we consider the following boundary value problem

$$
\begin{cases}A_{0} u:=-\Delta u=g & \text { in } D, \\ B u:=a\left(x^{\prime}\right) \frac{\partial u}{\partial \mathbf{n}}+b\left(x^{\prime}\right) u=\varphi & \text { on } \partial D .\end{cases}
$$

If we associate with problem (3.1) a continuous linear operator

$$
\mathcal{A}_{0}=\left(A_{0}, B\right): H^{2, p}(D) \longrightarrow L^{p}(D) \bigoplus B_{*}^{1-1 / p, p}(\partial D),
$$

then we have the following (see [27, Theorem 1.1]): 
Theorem 3.2. If condition (H.2) is satisfied, then the mapping $\mathcal{A}_{0}$ is an algebraic and topological isomorphism for all $1<p<\infty$.

In particular, we have, by Theorem 3.2,

$$
\text { ind } \mathcal{A}_{0}=0 \text {. }
$$

Step 2: If $C$ is the multiplication operator by the function $c(x) \in C(\bar{D})$, then it follows from an application of the Rellich and Kondrachov theorem (cf. [11, Theorem 7.26]) that the mapping

$$
\mathcal{C}: H^{2, p}(D) \longrightarrow L^{p}(D)
$$

is compact.

Therefore, we obtain that the mapping

$$
\mathcal{A}=\mathcal{A}_{0}+(\mathcal{C}, 0): H^{2, p}(D) \longrightarrow L^{p}(D) \bigoplus B_{*}^{1-1 / p, p}(\partial D)
$$

is a Fredholm operator with index zero, since we have, by assertion (3.2),

$$
\text { ind } \mathcal{A}=\text { ind } \mathcal{A}_{0}=0 \text {. }
$$

Step 3: On the other hand, the uniqueness result in Theorem 3.1 follows from a variant of the Bakel'man and Aleksandrov maximum principle in the framework of Sobolev spaces due to Bony [3, Théorème 2] (see also [29, Lemmas 3.25 and 3.26 and Theorem 3.27]):

Theorem 3.3 (The Weak Maximum Principle). Assume that condition (1.3) is satisfied. If a function $v \in H^{2, p}(D), N<p<\infty$, satisfies the condition

$$
A v(x) \leq 0 \quad \text { in } D
$$

then we have the inequality

$$
\max _{\bar{D}} v \leq \max _{\partial D} v^{+}
$$

where

$$
v^{+}(x)=\max \{v(x), 0\} .
$$

Theorem 3.4 (The Hopf Boundary Point Lemma). Assume that condition (1.3) is satisfied and that a function $v \in H^{2, p}(D), N<p<\infty$, satisfies the condition

$$
A v(x) \leq 0 \text { in } D .
$$

If $v(x)$ attains a strict local non-negative maximum at a point $x_{0}^{\prime}$ of $\partial D$, then we have

$$
\frac{\partial v}{\partial \mathbf{n}}\left(x_{0}^{\prime}\right)>0 .
$$

Theorem 3.5 (The Strong Maximum Principle). Assume that condition (1.3) is satisfied and that a function $v \in H^{2, p}(D), N<p<\infty$, satisfies the condition

$$
A v(x) \leq 0 \quad \text { in } D .
$$

If $v(x)$ attains a non-negative maximum at a point $x_{0}$ of $D$, then it is a constant. 
Here we recall that, for $N<p<\infty$,

$$
H^{2, p}(D) \subset C^{1}(\bar{D}) \text {. }
$$

By applying the maximum principle, we can obtain a uniqueness theorem for problem (1.2) in the framework of Sobolev spaces of $L^{p}$ style:

Corollary 3.6. Assume that conditions (H.2) and (1.3) are satisfied. If a function $u \in H^{2, p}(D), N<p<\infty$, satisfies the conditions

$$
\left\{\begin{array}{l}
A u=0 \quad \text { in } D, \\
B u=0 \quad \text { on } \partial D,
\end{array}\right.
$$

then it follows that $u(x) \equiv 0$ in $D$.

Proof. Assume, to the contrary, that $u(x) \not \equiv 0$ in $D$. Without loss of generality, we may assume that there exists a point $x_{0} \in \bar{D}$ such that

$$
u\left(x_{0}\right)=\max _{x \in \bar{D}} u(x)>0 .
$$
that

(a) If $x_{0} \in D$, then it follows from an application of Theorem 3.5 with $v:=-u$

$$
u(x) \equiv u\left(x_{0}\right)>0, \quad x \in D .
$$

Hence we have, for any point $x^{\prime} \in \partial D$,

$$
0=B u\left(x^{\prime}\right)=a\left(x^{\prime}\right) \frac{\partial u}{\partial \mathbf{n}}\left(x^{\prime}\right)+b\left(x^{\prime}\right) u\left(x^{\prime}\right)=b\left(x^{\prime}\right) u\left(x_{0}\right),
$$

and so

$$
u\left(x_{0}\right)=0,
$$

since $b\left(x^{\prime}\right) \not \equiv 0$ on $\partial D$. This is a contradiction.

(b) If $x_{0} \in \partial D$, then we may assume that $u(x)$ attains a strict positive maximum at a point $x_{0}$, that is,

$$
\left\{\begin{array}{l}
u\left(x_{0}\right)=\max _{x \in \bar{D}} u(x)>0, \\
u(x)<u\left(x_{0}\right), \quad x \in D .
\end{array}\right.
$$

Thus it follows from an application of Theorem 3.4 with $v:=u$ that

$$
\frac{\partial u}{\partial \mathbf{n}}\left(x_{0}\right)>0 \text {. }
$$

However, we have, by condition (H.2),

$$
0=B u\left(x_{0}\right)=a\left(x_{0}\right) \frac{\partial u}{\partial \mathbf{n}}\left(x_{0}\right)+b\left(x_{0}\right) u\left(x_{0}\right)>0 .
$$

This is also a contradiction. 
Step 4: Corollary 3.6 asserts that the mapping

$$
\mathcal{A}: H^{2, p}(D) \longrightarrow L^{p}(D) \bigoplus B_{*}^{1-1 / p, p}(\partial D)
$$

is injective for $N<p<\infty$. Hence it is also surjective for $N<p<\infty$, since ind $\mathcal{A}=0$.

Summing up, we have proved that the mapping

$$
\mathcal{A}=(A, B): H^{2, p}(D) \longrightarrow L^{p}(D) \bigoplus B_{*}^{1-1 / p, p}(\partial D)
$$

is an algebraic and topological isomorphism for $N<p<\infty$.

The proof of Theorem 3.1 (and hence Theorem 1.1) is complete.

\subsection{Positivity of the Resolvent}

In order to apply the Kreŭn and Rutman theorem (Theorem 2.1), we study the following homogeneous boundary value problem:

$$
\begin{cases}A u:=(-\Delta+c(x)) u=g & \text { in } D, \\ B u:=a\left(x^{\prime}\right) \frac{\partial u}{\partial \mathbf{n}}+b\left(x^{\prime}\right) u=0 & \text { on } \partial D .\end{cases}
$$

By applying Theorem 3.1, we find that problem (3.3) has a unique solution $u \in$ $H^{2, p}(D)$ for any $g \in L^{p}(D)$. Therefore, we can introduce a continuous linear operator (resolvent)

$$
R: L^{p}(D) \longrightarrow H^{2, p}(D)
$$

by the formula $u=R g$. Moreover, by the Ascoli and Arzelà theorem it follows that the resolvent $R$, considered as

$$
R: C(\bar{D}) \longrightarrow C^{1}(\bar{D})
$$

is compact if $N<p<\infty$. Indeed, it suffices to note that, by Sobolev's imbedding theorem, the space $H^{2, p}(D)$ is continuously imbedded into $C^{2-N / p}(\bar{D})$ with $2-$ $N / p>1$, for all $N<p<\infty$.

Now we introduce an ordered Banach subspace of $C(\bar{D})$ that combines the good properties of the resolvent $R$. To do this, we need the following:

Lemma 3.7. Assume that conditions (H.2) and (1.3) are satisfied. If $v(x) \in C(\bar{D})$ and if $v(x) \geq 0$ but $v(x) \not \equiv 0$ on $\bar{D}$, then the function $u=R v$ satisfies the following three conditions:

(a) $u\left(x^{\prime}\right)=0$ on $M=\left\{x^{\prime} \in \partial D: a\left(x^{\prime}\right)=0\right\}$.

(b) $u(x)>0$ on $\bar{D} \backslash M$.

(c) For the conormal derivative $\partial u / \partial \mathbf{n}$ of $u$, we have

$$
\frac{\partial u}{\partial \mathbf{n}}\left(x^{\prime}\right)<0 \quad \text { on } M .
$$

In particular, the resolvent $R: C(\bar{D}) \rightarrow C(\bar{D})$ is positive. 
Proof. (1) Since the function $u=R v \in H^{2, p}(D), N<p<\infty$, satisfies the condition

$$
A u=v \geq 0 \text { in } D,
$$

it follows from an application of the weak maximum principle (Theorem 3.3) that the function $u(x)$ may take its negative minimum only on the boundary $\partial D$.

However, we have the following:

Claim 3.1. The function $u=R v$ does not take its negative minimum on the boundary $\partial D$. In other words, the function $u(x)$ is non-negative on $\bar{D}$.

Proof. Assume, to the contrary, that there exists a point $x_{0}^{\prime} \in \partial D$ such that

$$
\left\{\begin{array}{l}
\min _{x \in \bar{D}} u(x)=u\left(x_{0}^{\prime}\right)<0, \\
u(x)>u\left(x_{0}^{\prime}\right), \quad x \in D .
\end{array}\right.
$$

Since the function $u=R v$ satisfies the condition

$$
A u(x)=v(x) \geq 0 \quad \text { in } D,
$$

it follows from an application of the boundary point lemma (Theorem 3.4) with $v:=-u$ that

Then we have, by condition (H.2),

$$
\frac{\partial u}{\partial \mathbf{n}}\left(x_{0}^{\prime}\right)<0 .
$$

$$
0=B u\left(x_{0}^{\prime}\right)=a\left(x_{0}^{\prime}\right) \frac{\partial u}{\partial \mathbf{n}}\left(x_{0}^{\prime}\right)+b\left(x_{0}^{\prime}\right) u\left(x_{0}^{\prime}\right)<0 .
$$

This is a contradiction.

(2) Furthermore, we have the following:

Claim 3.2. The function $u=R v$ is strictly positive in $D$.

Proof. Assume, to the contrary, that there exists a point $x_{0} \in D$ such that

$$
u\left(x_{0}\right)=0 .
$$

Then we obtain from the strong maximum principle (Theorem 3.5) that

$$
u(x) \equiv 0 \quad \text { in } D,
$$

so that

$$
v(x)=K u(x) \equiv 0 \quad \text { in } D .
$$

This contradicts the condition that $v(x) \not \equiv 0$ on $\bar{D}$.

(3) If there exists a point $x_{0}^{\prime} \in \partial D$ such that

$$
u\left(x_{0}^{\prime}\right)=0,
$$

then we have

$$
\begin{cases}A u(x)=v(x) \geq 0 & \text { in } D, \\ u\left(x_{0}^{\prime}\right)=\min _{x \in \bar{D}} u(x)=0, & \\ u(x)>0 & \text { in } D .\end{cases}
$$


Thus it follows from an application of the boundary point lemma (Theorem 3.4) with $v:=-u$ that

so that

$$
\frac{\partial u}{\partial \mathbf{n}}\left(x_{0}^{\prime}\right)<0,
$$

since we have

$$
a\left(x_{0}^{\prime}\right)=0,
$$

$$
0=B u\left(x_{0}^{\prime}\right)=a\left(x_{0}^{\prime}\right) \frac{\partial u}{\partial \mathbf{n}}\left(x_{0}^{\prime}\right)+b\left(x_{0}^{\prime}\right) u\left(x_{0}^{\prime}\right)=a\left(x_{0}^{\prime}\right) \frac{\partial u}{\partial \mathbf{n}}\left(x_{0}^{\prime}\right) .
$$

Conversely, if $a\left(x_{0}^{\prime}\right)=0$, then it follows that

$$
0=B u\left(x_{0}^{\prime}\right)=a\left(x_{0}^{\prime}\right) \frac{\partial u}{\partial \mathbf{n}}\left(x_{0}^{\prime}\right)+b\left(x_{0}^{\prime}\right) u\left(x_{0}^{\prime}\right)=b\left(x_{0}^{\prime}\right) u\left(x_{0}^{\prime}\right) .
$$

Hence we have, by condition (H.2),

$$
u\left(x_{0}^{\prime}\right)=0,
$$

since $b\left(x_{0}^{\prime}\right)>0$.

Summing up, we have proved that

$$
\begin{aligned}
& u\left(x^{\prime}\right)=0 \Longleftrightarrow a\left(x^{\prime}\right)=0 ; \\
& u(x)>0 \Longleftrightarrow x \in \bar{D} \backslash M .
\end{aligned}
$$

Finally, Assertion (c) is an immediate consequence of the boundary point lemma, since the function $u(x)$ attains its minimum 0 at the set $M$.

The proof of Lemma 3.7 is complete.

\subsection{The Ordered Banach Space $C_{e}(\bar{D})$}

Now we introduce an ordered Banach subspace $C_{e}(\bar{D})$ of $C(\bar{D})$ which combines the good properties of the operator $R$ with the good properties of the natural ordering of $C(\bar{D})$.

If we let

$$
e(x)=R 1(x)
$$

then it follows from an application of Theorem 3.1 that the function $e(x) \in$ $H^{2, p}(D), N<p<\infty$, is the unique solution of the problem

$$
\begin{cases}(-\Delta+c(x)) e=1 & \text { in } D \\ B e=0 & \text { on } \partial D .\end{cases}
$$

Moreover, it follows from an application of Lemma 3.7 with $v:=1$ that the function $e(x)=R 1(x)$ satisfies the conditions

$$
\begin{cases}e(x)>0 & \text { on } \bar{D} \backslash M, \\ e\left(x^{\prime}\right)=0 & \text { on } M, \\ \frac{\partial e}{\partial \mathbf{n}}\left(x^{\prime}\right)<0 & \text { on } M\end{cases}
$$


where

$$
M=\left\{x^{\prime} \in \partial D: a\left(x^{\prime}\right)=0\right\} .
$$

We define a subspace $C_{e}(\bar{D})$ of $C(\bar{D})$ by the formula

$$
\begin{aligned}
C_{e}(\bar{D})= & \{u \in C(\bar{D}): \text { there is a constant } \alpha>0 \text { such that } \\
& -\alpha e(x) \leq u(x) \leq \alpha e(x) \text { in } D\},
\end{aligned}
$$

with the norm

$$
\|u\|_{e}=\inf \{\alpha>0:-\alpha e(x) \leq u(x) \leq \alpha e(x) \text { in } D\} .
$$

If we let

$$
P_{e}=\left\{u \in C_{e}(\bar{D}): u \geq 0 \text { on } \bar{D}\right\},
$$

then it is easy to verify that the space $C_{e}(\bar{D})$ is an ordered Banach space having the positive cone $P_{e}$ with non-empty interior $\operatorname{Int}\left(P_{e}\right)$.

This setting has the advantages that it takes into consideration in an optimal way the a priori information given by the maximum principle and that it is amenable to the methods of abstract functional analysis (see [1], [12]). In fact, we have the following:

Proposition 3.8. The resolvent $R$ maps $C(\bar{D})$ compactly into $C_{e}(\bar{D})$. Moreover, the resolvent $R$, considered as an operator $R: C_{e}(\bar{D}) \rightarrow C_{e}(\bar{D})$, is strongly positive, that is, $R v \in \operatorname{Int}\left(P_{e}\right)$ for all $v \in P_{e} \backslash\{0\}$.

Proof. The proof is divided into three steps.

Step 1: By the positivity of $R$, it follows that $R$ maps $C(\bar{D})$ into $C_{e}(\bar{D})$. Indeed, since we have, for all $v \in C(\bar{D})$,

$$
-\|v\|_{C(\bar{D})} \leq v(x) \leq\|v\|_{C(\bar{D})} \quad \text { on } \bar{D}
$$

we obtain that

$$
-\|v\|_{C(\bar{D})} R 1(x) \leq R v(x) \leq\|v\|_{C(\bar{D})} R 1(x) \quad \text { on } \bar{D} .
$$

This proves that

$$
-c e(x) \leq R v(x) \leq c e(x) \text { in } D,
$$

with $c=\|v\|_{C(\bar{D})}$, that is, $R v \in C_{e}(\bar{D})$.

Step 2: Next we prove that $R: C(\bar{D}) \rightarrow C_{e}(\bar{D})$ is compact. To do this, we let

$$
C_{B}^{1}(\bar{D})=\left\{u \in C^{1}(\bar{D}): B u=0 \text { on } \partial D\right\} .
$$

Since $R$ maps $C(\bar{D})$ compactly into $C_{B}^{1}(\bar{D})$, it suffices to show that the inclusion mapping

$$
\iota: C_{B}^{1}(\bar{D}) \longrightarrow C_{e}(\bar{D})
$$

is continuous.

Step 2-a: We verify that $\iota$ maps $C_{B}^{1}(\bar{D})$ into $C_{e}(\bar{D})$. 
Let $u(x)$ be an arbitrary function in $C_{B}^{1}(\bar{D})$. Since we have, for some neighborhood $\omega$ of $M$ in $\partial D$,

it follows that

$$
\left\{\begin{array}{l}
b\left(x^{\prime}\right)>0 \quad \text { in } \omega \\
\frac{\partial e}{\partial \mathbf{n}}\left(x^{\prime}\right)<0 \quad \text { in } \omega
\end{array}\right.
$$

$$
\frac{u\left(x^{\prime}\right)}{e\left(x^{\prime}\right)}=\frac{\left(-\frac{a\left(x^{\prime}\right)}{b\left(x^{\prime}\right)}\right) \frac{\partial u}{\partial \mathbf{n}}\left(x^{\prime}\right)}{\left(-\frac{a\left(x^{\prime}\right)}{b\left(x^{\prime}\right)}\right) \frac{\partial e}{\partial \mathbf{n}}\left(x^{\prime}\right)}=\frac{\frac{\partial u}{\partial \mathbf{n}}\left(x^{\prime}\right)}{\frac{\partial e}{\partial \mathbf{n}}\left(x^{\prime}\right)} \quad \text { in } \omega \backslash M .
$$

Hence there exists a constant $c_{1}>0$ such that

$$
\left|u\left(x^{\prime}\right)\right| \leq c_{1} e\left(x^{\prime}\right) \quad \text { in } \omega .
$$

Thus, by using Taylor's formula we can find a neighborhood $W$ of $\omega$ in $D$ and a constant $c_{2}>0$ such that

$$
|u(x)| \leq c_{2} e(x) \quad \text { in } W .
$$

On the other hand, since we have, for some constant $\alpha>0$,

$$
e(x) \geq \alpha \text { on } \bar{D} \backslash W,
$$

we can find a constant $c_{3}>0$ such that

$$
\left|\frac{u(x)}{e(x)}\right| \leq c_{3} \quad \text { on } \bar{D} \backslash W .
$$

Therefore, there exists a constant $c>0$ such that

$$
-c e(x) \leq u(x) \leq c e(x) \text { on } \bar{D} .
$$

This proves that $u \in C_{e}(\bar{D})$.

Step 2-b: To prove assertion (3.4), we show that the inclusion mapping $\iota: C_{B}^{1}(\bar{D}) \rightarrow C_{e}(\bar{D})$ is closed. To do this, we assume that

$$
\begin{cases}u_{j} \in C_{B}^{1}(\bar{D}), & \\ u_{j} \longrightarrow u & \text { in } C_{B}^{1}(\bar{D}) \\ u_{j} \longrightarrow v & \text { in } C_{e}(\bar{D})\end{cases}
$$

Then there exists a sequence $\left\{c_{j}\right\}, c_{j} \rightarrow 0$, such that

$$
\left\|u_{j}-v\right\|_{C(\bar{D})} \leq c_{j}\|e\|_{C(\bar{D})} \text {. }
$$

This implies that $u_{j} \rightarrow v$ in $C(\bar{D})$, so that $u=v$.

Step 2-c: The continuity of $\iota$ follows from an application of the closed graph theorem.

Step 3: It remains to prove the strong positivity of $R$. 
Step 3-a: We show that, for any $v(x) \geq 0$ but $v(x) \not \equiv 0$ on $\bar{D}$, there exist constants $\beta>0$ and $\gamma>0$ such that

$$
\beta e(x) \leq R v(x) \leq \gamma e(x) \quad \text { on } \bar{D} .
$$

By the positivity of $R$, we may modify the function $v(x)$ in such a way that $v \in C^{1}(\bar{D})$. Furthermore, since the functions $u=R v$ and $e=R 1$ vanish only on the set $M$, it suffices to prove that there exists a neighborhood $W$ of $M$ in $D$ such that

$$
\beta e(x) \leq u(x) \text { in } W .
$$

We recall that, in a neighborhood $\omega$ of $M$ in $\partial D$,

$$
\begin{cases}u\left(x^{\prime}\right)=\left(-\frac{a\left(x^{\prime}\right)}{b\left(x^{\prime}\right)}\right) \frac{\partial u}{\partial \mathbf{n}}\left(x^{\prime}\right) & \text { in } \omega, \\ \frac{\partial u}{\partial \mathbf{n}}\left(x^{\prime}\right)<0 & \text { in } \omega\end{cases}
$$

and

$$
\begin{cases}e\left(x^{\prime}\right)=\left(-\frac{a\left(x^{\prime}\right)}{b\left(x^{\prime}\right)}\right) \frac{\partial e}{\partial \mathbf{n}}\left(x^{\prime}\right) & \text { in } \omega, \\ \frac{\partial e}{\partial \mathbf{n}}\left(x^{\prime}\right)<0 & \text { in } \omega .\end{cases}
$$

Thus we have, for $\beta$ sufficiently small,

$$
\begin{cases}u\left(x^{\prime}\right)-\beta e\left(x^{\prime}\right) \geq 0 & \text { in } \omega, \\ \frac{\partial}{\partial \mathbf{n}}(u-\beta e)\left(x^{\prime}\right)<0 & \text { in } \omega .\end{cases}
$$

Therefore, by using Taylor's formula we can find a neighborhood $W$ of $M$ in $D$ such that

$$
u(x)-\beta e(x) \geq 0 \quad \text { in } W .
$$

This proves estimate (3.6).

Step 3-b: Finally, we show that the function $u=R v$ is an interior point of the positive cone $P_{e}$.

If we take

$$
\varepsilon=\frac{\beta}{2},
$$

where $\beta$ is the same constant as in estimate (3.5), then, for all functions $w \in C_{e}(\bar{D})$ satisfying

we have, by estimate (3.5),

$$
\|w-R v\|_{e}<\varepsilon
$$

$$
w(x) \leq R v(x)+\varepsilon e(x) \leq(\gamma+\varepsilon) e(x) \quad \text { on } \bar{D},
$$

and also

$$
w(x) \geq R v(x)-\varepsilon e(x) \geq \frac{\beta}{2} e(x) \quad \text { on } \bar{D} .
$$

This implies that $w \in P_{e}$, that is, the function $R v$ is an interior point of $P_{e}$.

The proof of Proposition 3.8 is now complete. 
Now we consider the following non-homogeneous equation (similar to equation (2.1)): For a given function $h \in P_{e}$, find a function $u(x)$ such that

$$
\begin{cases}(-\Delta+c(x)-\lambda) u=h & \text { in } D \\ B u=0 & \text { on } \partial D .\end{cases}
$$

Then, combining Theorem 2.2 (with $K:=R$ and $\lambda:=1 / \lambda$ ) and Proposition 3.8 we obtain the main result of this subsection (see [12, Theorem 16.6]):

Theorem 3.9. If $r:=\lim _{n \rightarrow \infty} \sqrt[n]{\left\|R^{n}\right\|}$ is the principal eigenvalue of the operator $R$, then we have the following three assertions:

(i) If $0<\lambda<1 / r$, then equation (3.7) has a unique positive solution $u$ and $u \in \operatorname{Int}\left(P_{e}\right)$.

(ii) If $\lambda>1 / r$, then equation (3.7) has no positive solution.

(iii) If $\lambda=1 / r$, then equation (3.7) has no solution.

\subsection{Eigenvalues of the Resolvent $R$}

Now we consider the resolvent $R$ as an operator in the ordered Banach space $C_{e}(\bar{D})$, and prove important results concerning its eigenfunctions and corresponding eigenvalues.

First, Proposition 3.8 tells us that the resolvent

$$
R: C_{e}(\bar{D}) \longrightarrow C_{e}(\bar{D})
$$

is strongly positive and compact. Moreover, we find that all the eigenvalues of $R$ are positive. Indeed, if $\mu$ is an eigenvalue of $R$, that is, if we have

$$
R v=\mu v, \quad \mu \neq 0,
$$

then it follows that

$$
\left\{\begin{array}{l}
v \in H^{2, p}(D), \quad N<p<\infty, \\
A v=\frac{1}{\mu} v \text { in } D .
\end{array}\right.
$$

Hence, by applying Green's formula we obtain that

$$
\begin{aligned}
\frac{1}{\mu} \int_{D}|v(x)|^{2} d x & =(A v, v)_{L^{2}(D)} \\
& =-\int_{D} \Delta v(x) \cdot v(x) d x+\int_{D} c(x)|v(x)|^{2} d x \\
& =\int_{D}|\nabla v|^{2} d x+\int_{D} c(x)|v(x)|^{2} d x-\int_{\partial D} \frac{\partial v}{\partial \mathbf{n}} \cdot v d \sigma \\
& =\int_{D}|\nabla v|^{2} d x+\int_{\left\{a\left(x^{\prime}\right) \neq 0\right\}} \frac{b\left(x^{\prime}\right)}{a\left(x^{\prime}\right)} \cdot v^{2} d \sigma \\
& \geq 0
\end{aligned}
$$

This implies that

$$
\mu>0 \text {. }
$$


Therefore, we have proved that $R$ has a countable number of positive eigenvalues, $\mu_{j}$, which may accumulate only at 0 . Hence they may be arranged in a decreasing sequence

$$
\mu_{1} \geq \mu_{2} \geq \cdots \geq \mu_{j} \geq \cdots \longrightarrow 0,
$$

where each eigenvalue is repeated according to its multiplicity.

The next theorem, a sharper version of the Kreun and Rutman theorem (Theorem 2.1), characterizes the eigenvalues and positive eigenfunctions of the resolvent $R$ (cf. [14]):

Theorem 3.10. The resolvent $R$, considered as an operator $R: C_{e}(\bar{D}) \rightarrow C_{e}(\bar{D})$, has the following spectral properties:

(i) The largest eigenvalue $\mu_{1}$ is algebraically simple and has a positive eigenfunction $\psi_{1}(x)$.

(ii) No other eigenvalues, $\mu_{j}, j \geq 2$, have positive eigenfunctions.

\section{Proof of Theorem 1.2}

This section is devoted to the proof of Theorem 1.2 which is inspired by Brown and Lin [4, Theorem 3.5].

\subsection{Eigenvalue Problems with Indefinite Weight Function}

This subsection is devoted to the study of the eigenvalue problem (1.4) with indefinite weight function $m(x) \in C(\bar{D})$. First, we introduce a densely defined, selfadjoint operator $\mathfrak{A}$ from the Hilbert space $L^{2}(D)$ into itself as follows.

(a) The domain of definition $D(\mathfrak{A})$ is the space

$$
D(\mathfrak{A})=\left\{v \in H^{2,2}(D): B v:=a\left(x^{\prime}\right) \frac{\partial v}{\partial \mathbf{n}}+b\left(x^{\prime}\right) v=0 \text { on } \partial D\right\} .
$$

(b) $\mathfrak{A} v=-\Delta v, v \in D(\mathfrak{A})$.

Then the next theorem is a special case of Theorem 3.10 with $c(x) \equiv 0$ (cf. [23, Theorem 0]):

Theorem 4.1. Assume that condition (H.2) is satisfied. Then the spectrum of $\mathfrak{A}$ contains only the discrete eigenvalues

$$
0<\gamma_{1}<\gamma_{2} \leq \cdots
$$

The first eigenvalue $\gamma_{1}$ is algebraically simple and its corresponding eigenfunction $\varphi_{1}(x) \in C^{\infty}(\bar{D})$ may be chosen to be positive everywhere in D. Moreover, no other eigenvalues have positive eigenfunctions.

If we introduce a linear operator

$$
\mathcal{T}(\lambda)=\mathfrak{A}-\lambda m(x) I, \quad \lambda \geq 0
$$


then it follows that $\mathcal{T}(\lambda)$ is selfadjoint in $L^{2}(D)$ and further that the eigenvalues and eigenfunctions of $\mathcal{T}(\lambda)$ correspond to those of the problem

$$
\begin{cases}(-\Delta-\lambda m(x)) v=\mu v & \text { in } D \\ B v=0 & \text { on } \partial D .\end{cases}
$$

Furthermore, applying Theorem 3.10 to our situation we obtain the following:

Theorem 4.2. Assume that conditions (H.1) and (H.2) are satisfied. Then the spectrum of $\mathcal{T}(\lambda)$ contains only the discrete eigenvalues

$$
\mu_{1}(\lambda)<\mu_{2}(\lambda) \leq \cdots .
$$

The first eigenvalue $\mu_{1}(\lambda)$ is algebraically simple and its corresponding eigenfunction $\phi_{1}(x) \in H^{2, p}(D), N<p<\infty$, may be chosen to be positive everywhere in $D$.

Proof. Indeed, by rescaling we may assume that

$$
|m(x)|<1 \text { on } \bar{D} .
$$

Then it is easy to see that the eigenvalue problem (4.2) is equivalent to the eigenvalue problem

$$
\begin{cases}(-\Delta+\lambda(1-m(x))) v=(\mu+\lambda) v & \text { in } D \\ B v=0 & \text { on } \partial D\end{cases}
$$

where

$$
\lambda(1-m(x)) \geq 0 \quad \text { on } \bar{D} .
$$

By applying Theorem 3.10 with $c(x):=\lambda(1-m(x))$, we can obtain the following two assertions:

(i) The spectrum of problem (4.3) contains only the discrete eigenvalues

$$
0<\gamma_{1}(\lambda)<\gamma_{2}(\lambda) \leq \cdots .
$$

(ii) The first eigenvalue $\gamma_{1}(\lambda)$ is algebraically simple and its corresponding eigenfunction $\phi_{1}(x)$ may be chosen to be positive everywhere in $D$.

Therefore, Theorem 4.2 follows from two assertions (i) and (ii) by taking

$$
\mu_{j}(\lambda)=\gamma_{j}(\lambda)-\lambda, \quad j=1,2, \ldots
$$

\subsection{Proof of Theorem 1.2}

The proof of Theorem 1.2 is divided into six steps.

Step 1: If $\lambda \geq 0$, we let

$$
\begin{aligned}
Q_{\lambda}(v) & =(\mathcal{T}(\lambda) v, v)_{L^{2}(D)} \\
& =(\mathfrak{A} v, v)_{L^{2}(D)}-\lambda \int_{D} m(x) v^{2} d x \\
& =-\int_{D} \Delta v \cdot v d x-\lambda \int_{D} m(x) v^{2} d x, \quad v \in D(\mathfrak{A}) .
\end{aligned}
$$


Then the next lemma characterizes the range of possible eigenvalues corresponding to non-negative eigenfunctions:

Lemma 4.3. If there exists a non-negative eigenfunction $\psi(x)$ corresponding to an eigenvalue $\lambda$ of problem (1.4), then we have, for all $v \in D(\mathfrak{A})$,

$$
Q_{\lambda}(v) \geq 0 \text {. }
$$

Proof. (1) First, we prove the following:

Claim 4.1. The eigenfunctions $\phi_{j}(x)$ corresponding to the eigenvalues $\mu_{j}(\lambda), j \geq 2$, are orthogonal to the eigenfunction $\phi_{1}(x)$ corresponding to the eigenvalue $\mu_{1}(\lambda)$ :

$$
\int_{D} \phi_{1}(x) \phi_{j}(x) d x=0, \quad j \geq 2 .
$$

Proof. By Green's formula, it follows that

$$
\begin{aligned}
& \left(\mu_{1}(\lambda)-\mu_{j}(\lambda)\right) \int_{D} \phi_{1} \cdot \phi_{j} d x \\
= & \int_{D} \mathcal{T}(\lambda) \phi_{1} \cdot \phi_{j} d x-\int_{D} \phi_{1} \cdot \mathcal{T}(\lambda) \phi_{j} d x \\
= & \int_{D}(-\Delta-\lambda m(x)) \phi_{1} \cdot \phi_{j} d x-\int_{D} \phi_{1} \cdot(-\Delta-\lambda m(x)) \phi_{j} d x \\
= & -\int_{D} \Delta \phi_{1} \cdot \phi_{j} d x+\int_{D} \phi_{1} \cdot \Delta \phi_{j} d x \\
= & -\int_{\partial D} \frac{\partial \phi_{1}}{\partial \mathbf{n}} \cdot \phi_{j} d \sigma+\int_{\partial D} \phi_{1} \cdot \frac{\partial \phi_{j}}{\partial \mathbf{n}} d \sigma,
\end{aligned}
$$

where $d \sigma$ is the surface element of $\partial D$. However, note that the eigenfunctions $\phi_{1}(x)$ and $\phi_{j}(x)$ satisfy the boundary conditions

$$
\left(\begin{array}{ll}
\frac{\partial \phi_{1}}{\partial \mathbf{n}} & \phi_{1} \\
\frac{\partial \phi_{j}}{\partial \mathbf{n}} & \phi_{j}
\end{array}\right)\left(\begin{array}{l}
a\left(x^{\prime}\right) \\
b\left(x^{\prime}\right)
\end{array}\right)=\left(\begin{array}{l}
0 \\
0
\end{array}\right) \quad \text { on } \partial D
$$

Thus it follows that

$$
\left|\begin{array}{ll}
\frac{\partial \phi_{1}}{\partial \mathbf{n}} & \phi_{1} \\
\frac{\partial \phi_{j}}{\partial \mathbf{n}} & \phi_{j}
\end{array}\right|=0 \quad \text { on } \partial D
$$

since $\left(a\left(x^{\prime}\right), b\left(x^{\prime}\right)\right) \neq(0,0)$ on $\partial D$.

Therefore, we obtain from formula (4.6) that

$$
\begin{aligned}
\left(\mu_{1}(\lambda)-\mu_{j}(\lambda)\right) \int_{D} \phi_{1} \cdot \phi_{j} d x & =-\int_{\partial D} \frac{\partial \phi_{1}}{\partial \mathbf{n}} \cdot \phi_{j} d \sigma+\int_{\partial D} \phi_{1} \cdot \frac{\partial \phi_{j}}{\partial \mathbf{n}} d \sigma \\
& =0 .
\end{aligned}
$$

This proves formula (4.5), since $\mu_{1}(\lambda)-\mu_{j}(\lambda)<0, j \geq 2$. 
(2) If $\psi(x)$ is a non-negative eigenfunction corresponding to the eigenvalue $\lambda$ of problem (1.4), then it follows that $\psi(x)$ is an eigenfunction corresponding to the eigenvalue $\mu(\lambda)=0$ of problem (4.2). However, since $\phi_{1}(x)>0$ in $D$, we have

$$
\int_{D} \psi(x) \phi_{1}(x) d x>0
$$

In view of Claim 4.1, this implies that

$$
\mu_{1}(\lambda)=0 .
$$

On the other hand, by the spectrum theorem it follows that

$$
(\mathcal{T}(\lambda) v, v)_{L^{2}(D)} \geq \mu_{1}(\lambda)(v, v)_{L^{2}(D)}, \quad v \in D(\mathcal{T}(\lambda)) .
$$

Summing up, we obtain from assertions (4.7) and (4.8) that

$$
Q_{\lambda}(v)=(\mathfrak{A} v, v)_{L^{2}(D)}-\lambda \int_{D} m(x) v^{2} d x=(\mathcal{T}(\lambda) v, v)_{L^{2}(D)} \geq 0 .
$$

This proves assertion (4.4).

Step 2: Now we consider the Rayleigh quotient

$$
K(v)=\frac{(\mathfrak{A} v, v)_{L^{2}(D)}}{\int_{D} m(x) v^{2} d x}
$$

and let

$$
\lambda_{1}(m)=\inf \left\{K(v): v \in D(\mathfrak{A}), \int_{D} m(x) v^{2} d x>0\right\} .
$$

Since we have, by condition (H.2),

$$
a\left(x^{\prime}\right)=0 \Longrightarrow v\left(x^{\prime}\right)=0,
$$

it follows from an application of Green's formula that

$$
\begin{aligned}
(\mathfrak{A} v, v)_{L^{2}(D)} & =-\int_{D} \Delta v \cdot v d x \\
& =\int_{D}|\nabla v|^{2} d x-\int_{\partial D} \frac{\partial v}{\partial \mathbf{n}} \cdot v d \sigma \\
& =\int_{D}|\nabla v|^{2} d x+\int_{\left\{a\left(x^{\prime}\right) \neq 0\right\}} \frac{b\left(x^{\prime}\right)}{a\left(x^{\prime}\right)} \cdot v^{2} d \sigma,
\end{aligned}
$$

so that, by definition (4.9),

$$
\lambda_{1}(m) \geq 0 .
$$

More precisely, we have the following:

Lemma 4.4. The quantity $\lambda_{1}(m)$ can be estimated as follows:

$$
\lambda_{1}(m) \geq \frac{\gamma_{1}}{\left\|m^{+}\right\|_{L^{\infty}(D)}} .
$$

Here $\gamma_{1}>0$ is the first eigenvalue of the operator $\mathfrak{A}$, and

$$
m^{+}(x)=\max \{m(x), 0\}, \quad x \in D .
$$


Proof. By the spectrum theorem, it follows that

$$
(\mathfrak{A} v, v)_{L^{2}(D)} \geq \gamma_{1}(v, v)_{L^{2}(D)}, \quad v \in D(\mathfrak{A}) .
$$

If $v \in D(\mathfrak{A})$ satisfies the condition

$$
\int_{D} m(x) v^{2} d x>0
$$

then we find that

$$
K(v)=\frac{(\mathfrak{A} v, v)_{L^{2}(D)}}{\int_{D} m(x) v^{2} d x} \geq \frac{\gamma_{1}(v, v)_{L^{2}(D)}}{\int_{D} m(x) v^{2} d x} \geq \frac{\gamma_{1}}{\left\|m^{+}\right\|_{L^{\infty}(D)}} .
$$

By definition (4.9), this proves estimate (4.10).

Step 3: We begin by considering the case where $\lambda>\lambda_{1}(m)$ :

Lemma 4.5. If $\lambda>\lambda_{1}(m)$, then $\lambda$ is not an eigenvalue of problem (1.4) possessing a non-negative eigenfunction.

Proof. If $\lambda>\lambda_{1}(m)$, we can find a function $v \in D(\mathfrak{A})$ such that

$$
\begin{aligned}
& \int_{D} m(x) v^{2} d x>0 \\
& (\mathfrak{A} v, v)_{L^{2}(D)}<\lambda \int_{D} m(x) v^{2} d x .
\end{aligned}
$$

Hence we have

$$
Q_{\lambda}(v)=(\mathfrak{A} v, v)_{L^{2}(D)}-\lambda \int_{D} m(x) v^{2} d x<0 .
$$

Therefore, Lemma 4.5 follows from an application of Lemma 4.3.

Step 4: Next we consider the case where $0<\lambda<\lambda_{1}(m)$ :

Lemma 4.6. If $0<\lambda<\lambda_{1}(m)$, then we have, for all $v \in D(\mathfrak{A})$,

$$
Q_{\lambda}(v) \geq \gamma_{1}\left(1-\frac{\lambda}{\lambda_{1}(m)}\right)(v, v)_{L^{2}(D)} .
$$

Proof. If $0<\lambda<\lambda_{1}(m)$, we can write $Q_{\lambda}(v)$ in the form

$$
\begin{aligned}
Q_{\lambda}(v) & =(\mathfrak{A} v, v)_{L^{2}(D)}-\lambda \int_{D} m(x) v^{2} d x \\
& =\frac{\lambda}{\lambda_{1}(m)}(\mathfrak{A} v, v)_{L^{2}(D)}-\lambda \int_{D} m(x) v^{2} d x+\left(1-\frac{\lambda}{\lambda_{1}(m)}\right)(\mathfrak{A} v, v)_{L^{2}(D)} \\
& =\frac{\lambda}{\lambda_{1}(m)} Q_{\lambda_{1}(m)}(v)+\left(1-\frac{\lambda}{\lambda_{1}(m)}\right)(\mathfrak{A} v, v)_{L^{2}(D)}
\end{aligned}
$$

However, it is clear that

$$
(\mathfrak{A} v, v)_{L^{2}(D)} \geq \lambda_{1}(m) \int_{D} m(x) v^{2} d x, \quad v \in D(\mathfrak{A}),
$$


so that

$$
Q_{\lambda_{1}(m)}(v) \geq 0, \quad v \in D(\mathfrak{A}) .
$$

Therefore, we obtain from formula (4.13) and inequality (4.11) that

$$
Q_{\lambda}(v) \geq\left(1-\frac{\lambda}{\lambda_{1}(m)}\right)(\mathfrak{A} v, v)_{L^{2}(D)} \geq \gamma_{1}\left(1-\frac{\lambda}{\lambda_{1}(m)}\right)(v, v)_{L^{2}(D)} .
$$

This proves inequality (4.12).

Step 5: Combining Lemmas 4.5 and 4.6, we have the following:

Proposition 4.7. If $\lambda>0$ and $\lambda \neq \lambda_{1}(m)$, then $\lambda$ is not an eigenvalue of problem (1.4) possessing a non-negative eigenfunction.

Proof. By Lemma 4.5, it suffices to consider the case where $0<\lambda<\lambda_{1}(m)$.

If $0<\lambda<\lambda_{1}(m)$ and if there exists a non-negative eigenfunction $v(x)$ corresponding to $\lambda$ of problem (1.4), then we have

$$
\begin{cases}-\Delta v=\lambda m(x) v & \text { in } D \\ B v=0 & \text { on } \partial D\end{cases}
$$

This implies that

$$
\begin{aligned}
& v \in D(\mathfrak{A}) \\
& Q_{\lambda}(v)=(\mathfrak{A} v, v)_{L^{2}(D)}-\lambda \int_{D} m(x) v^{2} d x=0 .
\end{aligned}
$$

However, it follows from an application of Lemma 4.6 that

$$
0=Q_{\lambda}(v) \geq \gamma_{1}\left(1-\frac{\lambda}{\lambda_{1}(m)}\right)(v, v)_{L^{2}(D)},
$$

so that

$$
v=0 \text {. }
$$

This contradiction proves that if $0<\lambda<\lambda_{1}(m)$, then $\lambda$ is not an eigenvalue of problem (1.4) possessing a non-negative eigenfunction.

Step 6: Finally, the next theorem proves Theorem 1.2:

Theorem 4.8. Assume that condition (H.1) and (H.2) are satisfied. Then we have the following four assertions:

(i) $\lambda_{1}(m)$ is an eigenvalue of problem (1.4).

(ii) $\lambda_{1}(m)$ is algebraically simple.

(iii) $\lambda_{1}(m)$ admits a positive eigenfunction $\psi_{1}(x)$.

(iv) No other eigenvalues have positive eigenfunctions.

Proof. (1) We consider the following eigenvalue problem:

$$
\begin{cases}-\Delta w-\lambda_{1}(m) m(x) w=\mu w & \text { in } D \\ B w=0 & \text { on } \partial D\end{cases}
$$


Then it is easy to see that $\lambda_{1}(m)$ is an eigenvalue of problem (1.4) with corresponding eigenfunction $w(x)$ if and only if $\mu=0$ is an eigenvalue of problem (4.14) with corresponding eigenfunction $w(x)$.

To prove assertion (i), we introduce a densely defined, selfadjoint operator $\mathcal{S}: L^{2}(D) \rightarrow L^{2}(D)$ by the formula

$$
\mathcal{S}=\mathcal{T}\left(\lambda_{1}(m)\right)=\mathfrak{A}-\lambda_{1}(m) m(x) I .
$$

It suffices to show that the first eigenvalue $\mu_{1}\left(\lambda_{1}(m)\right)$ of the operator $\mathcal{S}$ is equal to zero, that is, $\mu_{1}\left(\lambda_{1}(m)\right)=0$ (see Figure 4.1).

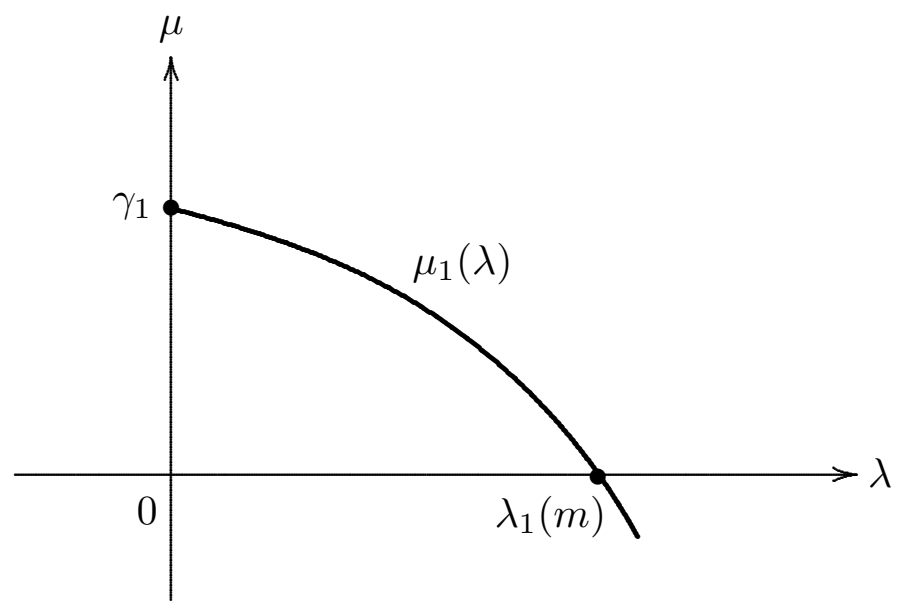

FIGURE 4.1

By the Rayleigh principle, it follows that

$$
\begin{aligned}
\mu_{1}\left(\lambda_{1}(m)\right) & =\inf \left\{\frac{(\mathcal{S} v, v)_{L^{2}(D)}}{\int_{D} v^{2} d x}: v \in D(\mathfrak{A})\right\} \\
& =\inf \left\{\frac{(\mathfrak{A} v, v)_{L^{2}(D)}-\lambda_{1}(m) \int_{D} m(x) v^{2} d x}{\int_{D} v^{2} d x}: v \in D(\mathfrak{A})\right\} .
\end{aligned}
$$

Since we have $Q_{\lambda_{1}(m)}(v) \geq 0$ for all $v \in D(\mathfrak{A})$, it follows from formula (4.15) that

$$
\mu_{1}\left(\lambda_{1}(m)\right) \geq 0 .
$$

The next claim proves assertion (i):

Claim 4.2. $\mu_{1}\left(\lambda_{1}(m)\right)=0$.

Proof. By definition (4.9) of $\lambda_{1}(m)$, we can find a sequence $\left\{v_{j}\right\} \subset D(\mathfrak{A})$ such that

$$
\begin{aligned}
& \int_{D} m(x) v_{j}^{2} d x=1, \\
& \left(\mathfrak{A} v_{j}, v_{j}\right)_{L^{2}(D)} \longrightarrow \lambda_{1}(m) \text { as } j \rightarrow \infty .
\end{aligned}
$$


Then we have

$$
Q_{\lambda_{1}(m)}\left(v_{j}\right)=\left(\mathfrak{A} v_{j}, v_{j}\right)_{L^{2}(D)}-\lambda_{1}(m) \int_{D} m(x) v_{j}^{2} d x \longrightarrow 0 \quad \text { as } j \rightarrow \infty .
$$

On the other hand, it follows that

$$
1=\int_{D} m(x) v_{j}^{2} d x \leq\left\|m^{+}\right\|_{L^{\infty}(D)} \int_{D} v_{j}^{2} d x,
$$

so that

$$
\int_{D} v_{j}^{2} d x \geq \frac{1}{\left\|m^{+}\right\|_{L^{\infty}(D)}} .
$$

Therefore, combining assertions (4.16) and (4.17) we obtain that

$$
\frac{Q_{\lambda_{1}(m)}\left(v_{j}\right)}{\int_{D} v_{j}^{2} d x} \longrightarrow 0 \quad \text { as } j \rightarrow \infty .
$$

By formula (4.15), this proves that $\mu_{1}\left(\lambda_{1}(m)\right)=0$.

(2) We recall that $\lambda_{1}(m)$ is an eigenvalue of problem (1.4) with corresponding eigenfunction $w(x)$ if and only if zero is an eigenvalue of the operator $\mathcal{S}$ with corresponding eigenfunction $w(x)$. However, Claim 4.2 tells us that zero is the first eigenvalue of $\mathcal{S}=\mathcal{T}\left(\lambda_{1}(m)\right)$. Therefore, assertions (ii) and (iii) follow from an application of Theorem 3.10 to our situation, just as in the proof of Theorem 4.2.

(3) Proposition 4.7 proves assertion (iv).

(4) Finally, the variational formula (1.5) is an immediate consequence of formula (4.9).

Now the proof of Theorem 1.2 is complete.

\section{The Comparison Theorem for First Eigenvalues}

In this section we study the inequalities among the first eigenvalues $\mu_{D}(\lambda), \mu_{N}(\lambda)$ and $\mu(\lambda)$ subject to Dirichlet, Neumann and general boundary conditions, respectively.

Step I: First, we consider the Dirichlet eigenvalue problem with an indefinite weight function $m(x) \in C^{\theta}(\bar{D})$ and a positive parameter $\lambda$

$$
\begin{cases}-\Delta \phi=\lambda m(x) \phi & \text { in } D \\ \phi=0 & \text { on } \partial D .\end{cases}
$$

The next theorem asserts the existence of the first positive eigenvalue of problem (5.1) (see [17], [7]):

Theorem 5.1. If the function $m(x) \in C^{\theta}(\bar{D})$ satisfies condition (H.1), then the first eigenvalue $\gamma_{1}(m)$ of the Dirichlet problem (5.1) is positive and algebraically simple, and its corresponding eigenfunction $\phi_{1}(x) \in C^{2+\theta}(\bar{D})$ may be chosen to be positive everywhere in D. Moreover, no other eigenvalues have positive eigenfunctions. 
If $v(x) \in C^{2+\theta}(\bar{D})$ is a positive eigenfunction corresponding to the first eigenvalue $\mu_{D}(\lambda)$ of the Dirichlet problem

$$
\begin{cases}(-\Delta-\lambda m(x)) v=\mu_{D}(\lambda) v & \text { in } D, \\ v=0 & \text { on } \partial D,\end{cases}
$$

then it is easy to see that $\lambda$ is the first eigenvalue $\gamma_{1}(m)$ of problem (5.1) with corresponding positive eigenfunction if and only if $\mu_{D}(\lambda)=0$ is an eigenvalue of problem (5.2) with corresponding positive eigenfunction.

Step II: Secondly, we consider the Neumann eigenvalue problem with an indefinite weight function $m(x) \in C^{\theta}(\bar{D})$ and a positive parameter $\lambda$

$$
\begin{cases}-\Delta \phi=\lambda m(x) \phi & \text { in } D, \\ \frac{\partial \phi}{\partial \mathbf{n}}=0 & \text { on } \partial D .\end{cases}
$$

The next theorem asserts the existence of the first eigenvalue of problem (5.3) (see [4, Theorem 3.13], [22, Theorems 2 and 3]):

Theorem 5.2. If the function $m(x) \in C^{\theta}(\bar{D})$ satisfies condition (H.3), then the Neumann problem (5.3) admits a unique non-negative eigenvalue $\nu_{1}(m)$ having a positive eigenfunction, and we have

$$
\begin{cases}\nu_{1}(m)>0 & \text { if } \int_{D} m(x) d x<0 \\ \nu_{1}(m)=0 & \text { if } \int_{D} m(x) d x \geq 0\end{cases}
$$

If $w(x) \in C^{2+\theta}(\bar{D})$ is a positive eigenfunction corresponding to the first eigenvalue $\mu_{N}(\lambda)$ of the Neumann problem

$$
\begin{cases}(-\Delta-\lambda m(x)) w=\mu_{N}(\lambda) w & \text { in } D \\ \frac{\partial w}{\partial \mathbf{n}}=0 & \text { on } \partial D\end{cases}
$$

then it is easy to see that $\lambda$ is the first eigenvalue $\nu_{1}(m)$ of problem (5.3) with corresponding positive eigenfunction if and only if $\mu_{N}(\lambda)=0$ is an eigenvalue of problem (5.4) with corresponding positive eigenfunction.

Step III: Thirdly, we consider the following eigenvalue problem:

$$
\begin{cases}(-\Delta-\lambda m(x)) u=\mu(\lambda) u & \text { in } D, \\ B u:=a\left(x^{\prime}\right) \frac{\partial u}{\partial \mathbf{n}}+b\left(x^{\prime}\right) u=0 & \text { on } \partial D .\end{cases}
$$

Then, by Claim 4.2 it follows that $\lambda$ is the first eigenvalue $\lambda_{1}(m)$ of problem (1.4) with corresponding positive eigenfunction if and only if $\mu(\lambda)=0$ is an eigenvalue of problem (5.5) with corresponding positive eigenfunction. It should be noticed that $\mu(\lambda)=\mu_{1}(\lambda)$ where $\mu_{1}(\lambda)$ is the first eigenvalue of problem (4.2).

The main result of this subsection is the following (see [12, Proposition 17.7]): 
Theorem 5.3. For all $\lambda \geq 0$, we have the inequalities (see Figure 5.1)

$$
\mu_{N}(\lambda)<\mu(\lambda)<\mu_{D}(\lambda) .
$$

In particular, it follows that

$$
\nu_{1}(m)<\lambda_{1}(m)<\gamma_{1}(m) .
$$

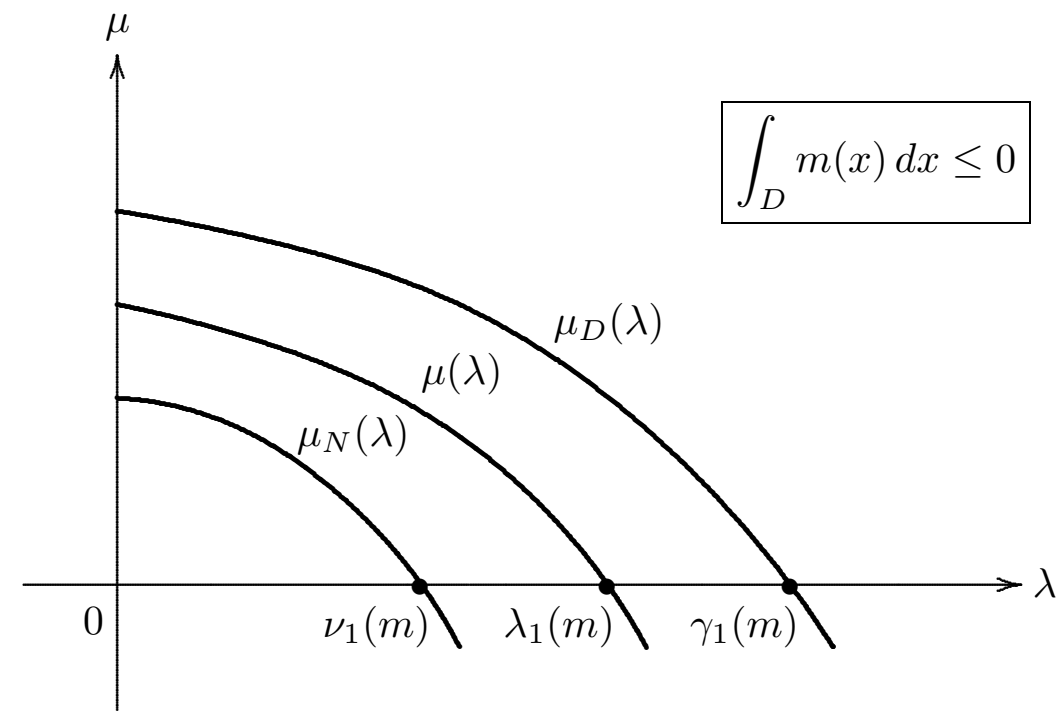

FiguRE 5.1

Proof. (1) First, we show that

$$
\mu_{D}(\lambda)>\mu(\lambda) .
$$

If we take a constant $c>0$ so large that

$$
\begin{aligned}
& c+\mu_{D}(\lambda)>0, \\
& c-\lambda m(x)>0 \text { in } D,
\end{aligned}
$$

then, by applying [27, Theorem 1.2] to our situation we can find a unique solution $u(x) \in C^{2+\theta}(\bar{D})$ of the problem

$$
\begin{cases}(-\Delta-\lambda m(x)+c) u=\left(\mu_{D}(\lambda)+c\right) v & \text { in } D \\ B u=0 & \text { on } \partial D .\end{cases}
$$

Here $v(x) \in C^{2+\theta}(\bar{D})$ is a positive eigenfunction of problem (5.2). By condition (5.7a), it follows from an application of the maximum principle (see Lemma 3.7) that

$$
u(x)>0 \text { in } D .
$$

Moreover, we have the following: 
Claim 5.1. $u(x) \geq v(x)$ in $D$.

Proof. Assume, to the contrary, that

$$
\alpha=\min _{\bar{D}}(u-v)<0 .
$$

Since we have

$$
\begin{cases}(-\Delta-\lambda m(x)+c) v=\left(\mu_{D}(\lambda)+c\right) v & \text { in } D \\ v>0 & \text { in } D \\ v=0 & \text { on } \partial D\end{cases}
$$

it follows that

$$
\begin{cases}(\Delta+\lambda m(x)-c)(u-v)=0 & \text { in } D, \\ u-v \geq 0 & \text { on } \partial D .\end{cases}
$$

This implies that the function $u(x)-v(x)$ may take its negative minimum $\alpha$ at an interior point of $D$. Thus, applying the strong maximum principle (Theorem 3.5) we obtain that

$$
u(x)-v(x) \equiv \alpha \quad \text { in } D .
$$

Hence we have, by condition (5.7b),

$$
0=(\Delta+\lambda m(x)-c)(u-v)=(\lambda m(x)-c) \alpha>0 \quad \text { in } D .
$$

This contradiction proves Claim 5.1.

By Claim 5.1, it follows that

$$
(-\Delta-\lambda m(x)+c) u=\left(\mu_{D}(\lambda)+c\right) v \leq\left(\mu_{D}(\lambda)+c\right) u \text { in } D .
$$

Hence we have

$$
\begin{cases}\left(\left(\mu_{D}(\lambda)+c\right)-(-\Delta-\lambda m(x)+c)\right) u \geq 0 & \text { in } D, \\ u>0 & \text { in } D, \\ B u=0 & \text { on } \partial D .\end{cases}
$$

Therefore, the desired assertion (5.6) follows by applying Theorem 3.9 with

$$
\begin{aligned}
\lambda & :=\mu_{D}(\lambda)+c, \\
r & :=\frac{1}{\mu(\lambda)+c} .
\end{aligned}
$$

(2) Next we show that

$$
\mu(\lambda)>\mu_{N}(\lambda)
$$

Let $u(x)$ be a positive eigenfunction corresponding to the first eigenvalue $\mu(\lambda)$ of problem (5.5). If we take a constant $d>0$ so large that

$$
\begin{aligned}
& d+\mu(\lambda)>0, \\
& d-\lambda m(x)>0 \quad \text { in } D,
\end{aligned}
$$


then we can find a unique solution $w(x) \in C^{2+\theta}(\bar{D})$ of the Neumann problem

$$
\begin{cases}(-\Delta-\lambda m(x)+d) w=(\mu(\lambda)+d) u & \text { in } D, \\ \frac{\partial w}{\partial \mathbf{n}}=0 & \text { on } \partial D .\end{cases}
$$

By the maximum principle, it follows that

$$
w(x)>0 \text { in } D .
$$

Moreover, we have the following:

Claim 5.2. $w(x) \geq u(x)$ in $D$.

Proof. Assume, to the contrary, that

$$
\beta=\min _{\bar{D}}(w-u)<0 .
$$

We remark that

$$
(\Delta+\lambda m(x)-d)(w-u)=0 \text { in } D .
$$

(a) If the function $w(x)-u(x)$ takes its negative minimum $\beta$ at an interior point $x_{0} \in D$, then, by applying the strong maximum principle (Theorem 3.5) we obtain that

$$
w(x)-u(x) \equiv \beta \quad \text { in } D .
$$

Hence we have, by condition $(5.9 \mathrm{~b})$,

$$
0=(\Delta+\lambda m(x)-d)(w-u)=(\lambda m(x)-d) \beta>0 \quad \text { in } D .
$$

This is a contradiction.

(b) If the function $w(x)-u(x)$ takes its negative minimum $\beta$ at a boundary point $x_{0}^{\prime} \in \partial D$, then, by applying the boundary point lemma (Theorem 3.4) we obtain that

This implies that

$$
\frac{\partial w}{\partial \mathbf{n}}\left(x_{0}^{\prime}\right)-\frac{\partial u}{\partial \mathbf{n}}\left(x_{0}^{\prime}\right)=\frac{\partial(w-u)}{\partial \mathbf{n}}\left(x_{0}^{\prime}\right)<0 .
$$

since we have

$$
\frac{\partial u}{\partial \mathbf{n}}\left(x_{0}^{\prime}\right)>0,
$$

On the other hand, we have

$$
\frac{\partial w}{\partial \mathbf{n}}=0 \quad \text { on } \partial D
$$

$$
0=B u\left(x_{0}^{\prime}\right)=a\left(x_{0}^{\prime}\right) \frac{\partial u}{\partial \mathbf{n}}+b\left(x_{0}^{\prime}\right) u\left(x_{0}^{\prime}\right) .
$$

However, it follows from condition (H.2) that

$$
a\left(x_{0}^{\prime}\right)>0 .
$$

Indeed, it suffices to note that if $a\left(x_{0}^{\prime}\right)=0$, then we have, by condition (H.2),

$$
u\left(x_{0}^{\prime}\right)=0,
$$


and so

$$
0 \leq w\left(x_{0}^{\prime}\right)=w\left(x_{0}^{\prime}\right)-u\left(x_{0}^{\prime}\right)=\beta<0 .
$$

This is a contradiction.

Therefore, combining assertions (5.10) and (5.11) we find that

$$
0<\frac{\partial u}{\partial \mathbf{n}}\left(x_{0}^{\prime}\right)=-\frac{b\left(x_{0}^{\prime}\right)}{a\left(x_{0}^{\prime}\right)} u\left(x_{0}^{\prime}\right) \leq 0 .
$$

This contradiction proves Claim 5.2.

By Claim 5.2, it follows that

$$
(-\Delta-\lambda m(x)+d) w=(\mu(\lambda)+d) u \leq(\mu(\lambda)+d) w \text { in } D .
$$

Hence we have

$$
\begin{cases}((\mu(\lambda)+d)-(-\Delta-\lambda m(x)+d)) w \geq 0 & \text { in } D \\ w>0 & \text { in } D \\ \frac{\partial w}{\partial \mathbf{n}}=0 & \text { on } \partial D\end{cases}
$$

Therefore, the desired assertion (5.8) follows by applying Theorem 3.9 with

$$
\begin{aligned}
& \lambda:=\mu(\lambda)+d, \\
& r:=\frac{1}{\mu_{N}(\lambda)+d} .
\end{aligned}
$$

Now the proof of Theorem 5.3 is complete.

\section{Proof of Theorem $1.3-(1)$}

This section and the next section are devoted to the proof of Theorem 1.3. Our approach to problem (1.1) is a modification of that of Ouyang [18] adapted to the present context.

Step I: First, we begin with the following lower bound on the parameter $\lambda$ for the existence of positive solutions of problem (1.1):

Lemma 6.1. Assume that conditions (H.1) and (H.2) are satisfied. If there exists a positive solution $u(\lambda) \in C^{2}(\bar{D})$ of problem (1.1) for $\lambda>0$, then we have the inequality

$$
\lambda>\lambda_{1}(m) .
$$

Proof. Let $\psi_{1}(x)$ be a positive eigenfunction corresponding to the first eigenvalue $\lambda_{1}(m)$ :

$$
\begin{cases}-\Delta \psi_{1}=\lambda_{1}(m) m(x) \psi_{1} & \text { in } D \\ \psi_{1}>0 & \text { in } D \\ B \psi_{1}=0 & \text { on } \partial D\end{cases}
$$


Then it follows from an application of Green's formula that

$$
\begin{aligned}
0= & \int_{D}\left(\Delta u(\lambda)+\lambda m(x) u(\lambda)-\lambda h(x) u(\lambda)^{2}\right) \psi_{1} d x \\
= & \int_{D} u(\lambda) \cdot \Delta \psi_{1} d x+\lambda \int_{D} m(x) u(\lambda) \psi_{1} d x-\lambda \int_{D} h(x) u(\lambda)^{2} \psi_{1} d x \\
& +\int_{\partial D} \frac{\partial u(\lambda)}{\partial \mathbf{n}} \psi_{1} d \sigma-\int_{\partial D} u(\lambda) \frac{\partial \psi_{1}}{\partial \mathbf{n}} d \sigma \\
= & \left(\lambda-\lambda_{1}(m)\right) \int_{D} m(x) u(\lambda) \psi_{1} d x-\lambda \int_{\partial D} h(x) u(\lambda)^{2} \psi_{1} d x \\
& +\int_{\partial D}\left(\frac{\partial u(\lambda)}{\partial \mathbf{n}} \psi_{1}-u(\lambda) \frac{\partial \psi_{1}}{\partial \mathbf{n}}\right) d \sigma .
\end{aligned}
$$

However, we recall that the functions $u(\lambda)$ and $\psi_{1}$ satisfy the boundary conditions

$$
\left(\begin{array}{cc}
\frac{\partial u(\lambda)}{\partial \mathbf{n}} & u(\lambda) \\
\frac{\partial \psi_{1}}{\partial \mathbf{n}} & \psi_{1}
\end{array}\right)\left(\begin{array}{l}
a\left(x^{\prime}\right) \\
b\left(x^{\prime}\right)
\end{array}\right)=\left(\begin{array}{l}
0 \\
0
\end{array}\right) \quad \text { on } \partial D
$$

so that

$$
\left|\begin{array}{ll}
\frac{\partial u(\lambda)}{\partial \mathbf{n}} & u(\lambda) \\
\frac{\partial \psi_{1}}{\partial \mathbf{n}} & \psi_{1}
\end{array}\right|=0 \quad \text { on } \partial D
$$

since $\left(a\left(x^{\prime}\right), b\left(x^{\prime}\right)\right) \neq(0,0)$ on $\partial D$.

Therefore, we obtain from formula (6.2) that

$$
\left(\lambda-\lambda_{1}(m)\right) \int_{D} m(x) u(\lambda) \psi_{1} d x-\lambda \int_{D} h(x) u(\lambda)^{2} \psi_{1} d x=0
$$

so that

$$
\lambda-\lambda_{1}(m)=\frac{\lambda \int_{D} h(x) u(\lambda)^{2} \psi_{1} d x}{\int_{D} m(x) u(\lambda) \psi_{1} d x}>0 .
$$

This proves the lower bound (6.1).

Step II: Conversely, we construct a positive solution $u(\lambda)$ of problem (1.1) for every $\lambda>\lambda_{1}(m)$.

By using the operator $R$ for problem (1.2) with $c(x):=0$, we transform problem (1.1) into a nonlinear operator equation in the ordered Banach space $C_{e}(\bar{D})$ (see [1]). It follows from an application of Proposition 3.8 that a function $u(x)$ is a solution of problem (1.1) if and only if it satisfies the equation

$$
u=\lambda R\left(m(x) u-h(x) u^{2}\right) \quad \text { in } C_{e}(\bar{D}) .
$$

Moreover, just as in the proof of Hess and Kato [13, Theorem 2] we extend the function

$$
f(x, s)=m(x) s-h(x) s^{2}
$$


as an odd function in the variable $s$ as follows:

$$
\tilde{f}(x, s)= \begin{cases}m(x) s-h(x) s^{2} & \text { if } s>0, \\ m(x) s+h(x) s^{2} & \text { if } s \leq 0 .\end{cases}
$$

Then we associate with the function $\tilde{f}(x, s)$ the Nemytskii operator $\widetilde{F}(u)$ defined by the formula

$$
\widetilde{F}(u)=\tilde{f}(x, u(x)), \quad x \in \bar{D},
$$

and consider instead of equation (6.3) the following equation:

$$
u=\lambda R(\widetilde{F}(u)) \quad \text { in } C_{e}(\bar{D}) .
$$

We notice that $u(x)$ is a solution of equation (6.4) if and only if $-u(x)$ is a solution; hence we may identify positive solutions with negative solutions in what follows.

The proof of Theorem 1.3 is based on the following bifurcation theorem from a simple eigenvalue due to Crandall and Rabinowitz [6, Theorem 1.7]:

Theorem 6.2. Let $f(\lambda, x)$ be a $C^{k}$ map, $k \geq 3$, of a neighborhood of $\left(\lambda_{1}, 0\right)$ in a Banach space $\mathbf{R} \times \mathcal{X}$ into a Banach space $\mathcal{Y}$ such that

$$
f\left(\lambda_{1}, 0\right)=0 .
$$

Assume that the following four conditions are satisfied:

(i) $f_{\lambda}\left(\lambda_{1}, 0\right)=0$.

(ii) The null space $N\left(f_{x}\left(\lambda_{1}, 0\right)\right)$ is one dimensional, spanned by a vector $x_{0}$.

(iii) The range $R\left(f_{x}\left(\lambda_{1}, 0\right)\right)$ has codimension one in the space $\mathcal{Y}$.

(iv) $f_{\lambda \lambda}\left(\lambda_{1}, 0\right) \in R\left(f_{x}\left(\lambda_{1}, 0\right)\right)$ and $f_{\lambda x}\left(\lambda_{1}, 0\right) x_{0} \notin R\left(f_{x}\left(\lambda_{1}, 0\right)\right)$.

Then the point $\left(\lambda_{1}, 0\right)$ is a bifurcation point for the equation $f(\lambda, x)=0$. In fact, the set of solutions of $f(\lambda, x)=0$ near $\left(\lambda_{1}, 0\right)$ consists of two $C^{k-2}$ curves $\Gamma_{1}$ and $\Gamma_{2}$ intersecting only at the point $\left(\lambda_{1}, 0\right)$. Furthermore, the curve $\Gamma_{1}$ is tangent to the $\lambda$-axis at $\left(\lambda_{1}, 0\right)$ and may be parametrized by $\lambda$ as

$$
\Gamma_{1}=\left\{\left(\lambda, x_{1}(\lambda)\right):\left|\lambda-\lambda_{1}\right|<\varepsilon\right\},
$$

while the curve $\Gamma_{2}$ may be parametrized by a variable $s$ as

$$
\Gamma_{2}=\left\{\left(\lambda_{2}(s), s x_{0}+x_{2}(s)\right):|s|<\varepsilon\right\} .
$$

Here

$$
x_{2}(0)=\frac{d x_{2}}{d s}(0)=0, \quad \lambda_{2}(0)=\lambda_{1} .
$$


We shall apply Theorem 6.2 with

$$
\begin{aligned}
& \mathcal{X}=\mathcal{Y}:=C_{e}(\bar{D}), \\
& f(\lambda, x):=u-\lambda R F(u)=u-\lambda R\left(m(x) u-h(x) u^{2}\right), \\
& f_{x}\left(\lambda_{1}(m), 0\right):=I-\lambda_{1}(m) R M, \\
& f_{t x}\left(\lambda_{1}(m), 0\right):=-R M, \\
& \lambda_{1}:=\lambda_{1}(m), \\
& x_{0}:=\psi_{1}(x) .
\end{aligned}
$$

Step II-a: First, the next lemma proves the existence of positive solutions of problem (1.1) emanating from the point $\left(\lambda_{1}(m), 0\right)$ :

Lemma 6.3. Assume that conditions (H.1) and (H.2) are satisfied. Then there exists a positive bifurcation solution curve $(\lambda, u(\lambda))$ of problem (1.1) starting at the point $\left(\lambda_{1}(m), 0\right)$.

Proof. (1) The Crandall and Rabinowitz local bifurcation theorem (Theorem 6.2) may be employed to assert that the simplicity of the eigenvalue $\lambda_{1}(m)$ guarantees the existence of the continuum of non-trivial solutions of problem (1.1) emanating from the point $\left(\lambda_{1}(m), 0\right)$, which can be expressed as the union of two subcontinua intersecting at the point $\left(\lambda_{1}(m), 0\right)$.

To do this, it suffices to verify the following two assertions:

(1a) $\operatorname{dim} N\left(I-\lambda_{1}(m) R M\right)=\operatorname{codim} R\left(I-\lambda_{1}(m) R M\right)=1$.

(1b) $R M \psi_{1} \notin R\left(I-\lambda_{1}(m) R M\right)$.

Proof of Assertion (1a): First, since the operator

$$
R M: C_{e}(\bar{D}) \longrightarrow C(\bar{D}) \longrightarrow C_{e}(\bar{D})
$$

is compact, applying the Riesz and Schauder theory [30, Chapter X, Section 5, Theorem 3] we obtain that the index of the operator

$$
I-\lambda_{1}(m) R M: C_{e}(\bar{D}) \longrightarrow C_{e}(\bar{D})
$$

is equal to zero, that is,

$$
\operatorname{ind}\left(I-\lambda_{1}(m) R M\right)=0 .
$$

However, Theorem 1.2 tells us that the null space

$$
N\left(I-\lambda_{1}(m) R M\right)=N\left(-\Delta-\lambda_{1}(m) M\right)
$$

is one dimensional, spanned by the positive eigenfunction $\psi_{1}(x)$. In particular, we have

$$
\operatorname{dim} N\left(I-\lambda_{1}(m) R M\right)=1 .
$$

Therefore, assertion (1a) follows by combining assertions (6.5) and (6.6).

Proof of Assertion (1b): Secondly, we assume, to the contrary, that

$$
R M \psi_{1} \in R\left(I-\lambda_{1}(m) R M\right),
$$


or equivalently,

$$
M \psi_{1}=\frac{1}{\lambda_{1}(m)} \Delta \psi_{1} \in R\left(-\Delta-\lambda_{1}(m) M\right) .
$$

Then we have, by Green's formula,

$$
\begin{aligned}
0 & =\left(M \psi_{1}, \psi_{1}\right)_{L^{2}(D)} \\
& =\frac{1}{\lambda_{1}(m)}\left(-\Delta \psi_{1}, \psi_{1}\right)_{L^{2}(D)} \\
& =\frac{1}{\lambda_{1}(m)}\left\{\int_{D}\left|\nabla \psi_{1}\right|^{2} d x+\int_{\left\{a\left(x^{\prime}\right) \neq 0\right\}} \frac{b\left(x^{\prime}\right)}{a\left(x^{\prime}\right)} \cdot \psi_{1}^{2} d \sigma\right\} .
\end{aligned}
$$

This implies that

$$
\nabla \psi_{1}(x) \equiv 0 \quad \text { in } D
$$

and that

$$
\psi_{1}\left(x^{\prime}\right)=0 \quad \text { if } b\left(x^{\prime}\right) \neq 0 .
$$

Therefore, we obtain from condition (H.2) that

$$
\psi_{1}(x) \equiv 0 \quad \text { in } D
$$

This contradiction proves assertion (1b).

(2) Moreover, by using the maximum principle we find that these subcontinua are locally the strictly positive and the strictly negative solutions of problem (1.1) as in Figure 6.1.

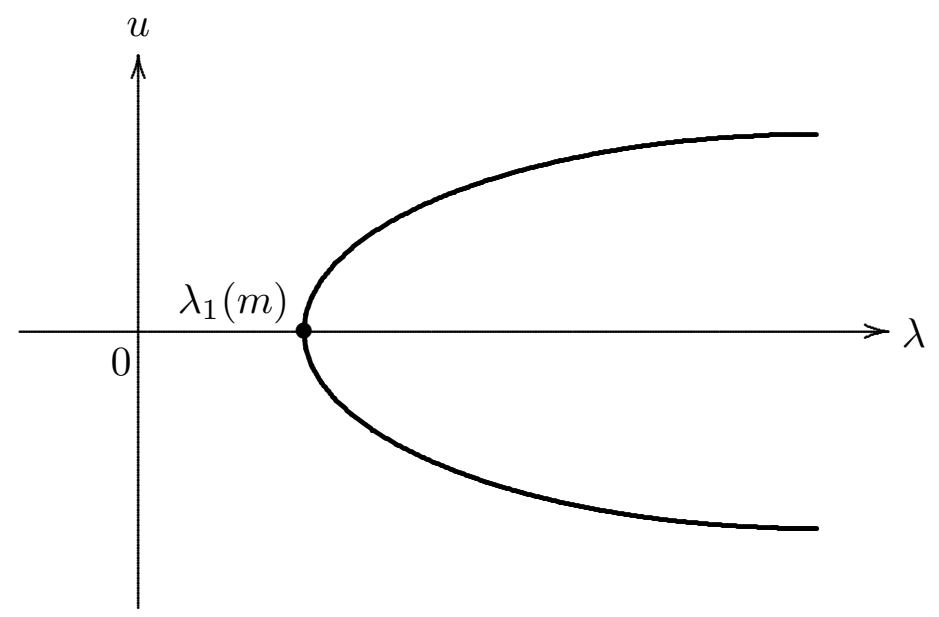

Figure 6.1 
Indeed, assume, to the contrary, that there exists a sequence $\left(\lambda_{j}, u_{j}\right)$, with $\lambda_{j}>0$ and $u_{j} \in C_{e}(\bar{D})$, such that

$$
\begin{aligned}
& u_{j}=\lambda_{j} R\left(\widetilde{F}\left(u_{j}\right)\right), \\
& \lambda_{j} \rightarrow \lambda_{1}(m), \\
& u_{j} \rightarrow 0 \quad \text { in } C_{e}(\bar{D}), \\
& u_{j} \notin \operatorname{Int}\left(P_{e}\right) .
\end{aligned}
$$

If we let

$$
v_{j}=\frac{u_{j}}{\left\|u_{j}\right\|_{e}}
$$

then it follows that

$$
\begin{aligned}
& v_{j} \notin \operatorname{Int}\left(P_{e}\right), \\
& \left\|v_{j}\right\|_{e}=1,
\end{aligned}
$$

and

$$
v_{j}=\lambda_{j} \frac{R\left(\widetilde{F}\left(u_{j}\right)\right)}{\left\|u_{j}\right\|_{e}} .
$$

By the compactness of $R: C_{e}(\bar{D}) \rightarrow C_{e}(\bar{D})$, we may choose a subsequence, denoted again by $\left\{v_{j}\right\}$, which converges to some function $v$ in $C_{e}(\bar{D})$. Therefore, passing to the limit in formula (6.7) we obtain that

$$
\begin{gathered}
v \notin \operatorname{Int}\left(P_{e}\right), \\
\|v\|_{e}=1,
\end{gathered}
$$

and that

$$
v=\lambda_{1}(m) R(m(x) v)
$$

This implies that

$$
v \in C^{2+\theta}(\bar{D})
$$

and

$$
\begin{cases}-\Delta v=\lambda_{1}(m) m(x) v & \text { in } D, \\ B v=0 & \text { on } \partial D .\end{cases}
$$

Therefore, we arrive at a contradiction (perhaps by changing sign in $v$ )

$$
v \in \operatorname{Int}\left(P_{e}\right)
$$

since $\lambda_{1}(m)$ is a algebraically simple eigenvalue of problem (1.4) having a positive eigenfunction in $\operatorname{Int}\left(P_{e}\right)$.

(3) We show that these subcontinua are globally the strictly positive and the strictly negative solutions of problem (1.1). 
Indeed, assume, to the contrary, that there exists a point $\left(\lambda_{0}, u_{0}\right)$ such that

$$
\begin{aligned}
& \lambda_{0}>0, \\
& u_{0} \in \partial\left(P_{e}\right), \\
& u_{0}>0, \\
& u_{0}=\lambda_{0} R\left(\widetilde{F}\left(u_{0}\right)\right) .
\end{aligned}
$$

If we let

then it follows that

$$
c=\max _{\substack{x \in \bar{D} \\ 0 \leq s \leq\left\|u_{0}\right\|_{C(\bar{D})}}}|m(x)-h(x) s|+1,
$$

$$
\begin{aligned}
\left(-\Delta+\lambda_{0} c\right) u_{0} & =\lambda_{0}\left(\widetilde{F}\left(u_{0}\right)+c u_{0}\right) \\
& =\lambda_{0} u_{0}\left(m(x)-h(x) u_{0}+c\right)>0 \quad \text { in } D .
\end{aligned}
$$

Therefore, by the maximum principle (cf. Proposition 3.8) we arrive at a contradiction

$$
u_{0} \in \operatorname{Int}\left(P_{e}\right) .
$$

(4) Finally, the Rabinowitz global bifurcation theorem [20, Theorem 1.10] tells us that the subcontinuum $\mathcal{C}$ of positive solutions emanating from $\left(\lambda_{1}(m), 0\right)$ is either unbounded or contains another bifurcation point $\left(\lambda_{0}, 0\right)$ with $\lambda_{0} \neq \lambda_{1}(m)$ (cf. [8, Theorem 29.2]).

However, just as in step (3) we can prove that the subcontinuum $\mathcal{C}$ can not contain a point $\left(\lambda_{0}, 0\right)$ with $\lambda_{0} \neq \lambda_{1}(m)$; hence $\mathcal{C}$ must be unbounded.

The proof of Lemma 6.3 is complete.

Step II-b: Secondly, the next lemma proves the existence of a critical value $\bar{\lambda}(h) \in\left(\lambda_{1}(m),+\infty\right]$ such that we can parametrize the positive bifurcation solution curve $(\lambda, u(\lambda))$ by $\lambda, \lambda_{1}(m)<\lambda<\bar{\lambda}(h)$, as a $C^{1}$ curve as in Figure 6.2.

Lemma 6.4. There exists a constant $\lambda^{*} \in\left(\lambda_{1}(m),+\infty\right]$ such that we have a positive solution $(\lambda, u(\lambda))$ of the equation $u=H(\lambda, u)$ for all $\lambda \in\left(\lambda_{1}(m), \lambda^{*}\right)$.

Proof. First, we introduce a mapping

$$
H(\lambda, v): \mathbf{R}^{+} \times C_{e}(\bar{D}) \longrightarrow C_{e}(\bar{D})
$$

defined by the formula

$$
H(\lambda, v)=\lambda(\lambda-\Delta)^{-1}(F(v)+v), \quad \lambda>0, v \in C_{e}(\bar{D}) .
$$

Then it is easy to see that

$$
-\Delta u=\lambda F(u)
$$

if and only if

$$
u=H(\lambda, u) \quad \text { in } C_{e}(\bar{D})
$$

By rescaling, we may assume that

$$
m(x)-2 h(x) s+1>0, \quad x \in \bar{D}, 0 \leq s \leq\|u\|_{C(\bar{D})}+1 .
$$




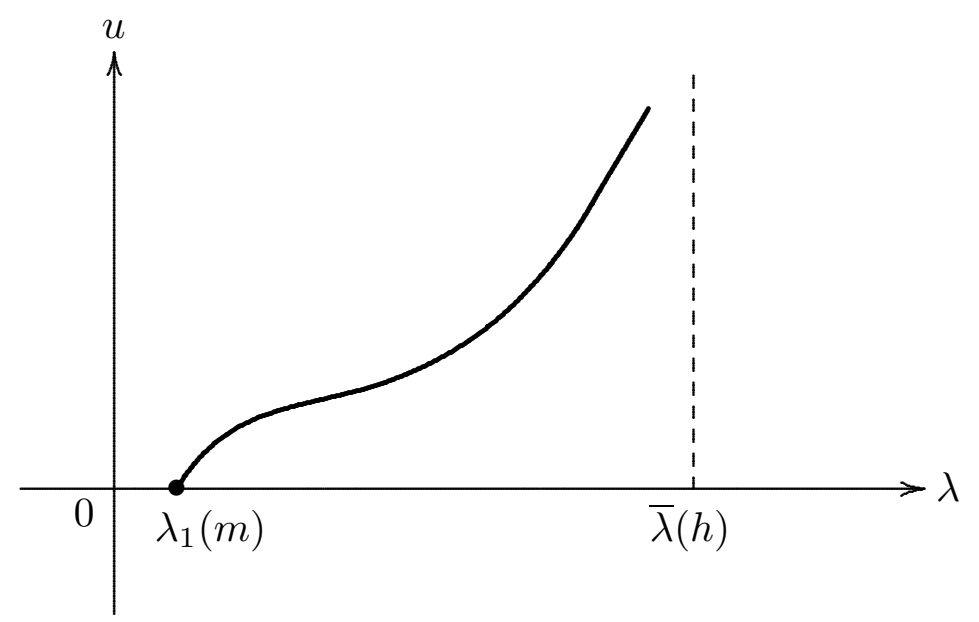

Figure 6.2

Then, applying Proposition 3.8 to our situation we obtain that the Fréchet derivative

$$
H_{v}(\lambda, u)=\lambda(\lambda-\Delta)^{-1}\left(F^{\prime}(u)+I\right): C_{e}(\bar{D}) \longrightarrow C_{e}(\bar{D})
$$

at $(\lambda, u)$ is strongly positive and compact.

The next claim guarantees the bijectivity of the Fréchet derivative $H_{v}(\lambda, u)$ :

Claim 6.1. If $r^{*}=\operatorname{spr}\left(H_{v}(\lambda, u)\right)$ is the principal eigenvalue of $H_{v}(\lambda, u)$, then it follows that $0<r^{*}<1$.

Proof. Assume, to the contrary, that

$$
r^{*} \geq 1
$$

By the Kreun and Rutman theorem (Theorem 2.1), it follows that there exists a function $w \in \operatorname{Int}\left(P_{e}\right)$ such that

$$
H_{v}(\lambda, u) w=r^{*} w .
$$

However, we can find a constant $t_{0}>0$ such that

$$
u-t_{0} r^{*} w \in \partial P_{e},
$$

since $u, w \in \operatorname{Int}\left(P_{e}\right)$. Then we have

$$
H\left(\lambda, u-t_{0} r^{*} w\right) \in P_{e} .
$$

Indeed, it suffices to note that the function $H(\lambda, \cdot)$ is increasing and $H(\lambda, 0)=0$. 
On the other hand, it follows that

$$
\begin{aligned}
& u-t_{0} r^{*} w \\
= & H(\lambda, u)-t_{0} H_{v}(\lambda, u) w \\
= & \lambda(\lambda-\Delta)^{-1}\left(\left(m(x) u-h(x) u^{2}+u\right)-t_{0}(m(x)-2 h(x) u+1) w\right) \\
= & H\left(\lambda, u-t_{0} w\right)+\lambda t_{0}^{2}(\lambda-\Delta)^{-1}\left(h(x) w^{2}\right) \\
\geq & H\left(\lambda, u-t_{0} r^{*} w\right)+\lambda t_{0}^{2}(\lambda-\Delta)^{-1}\left(h(x) w^{2}\right),
\end{aligned}
$$

since $u-t_{0} w \geq u-t_{0} r^{*} w$ for $r^{*} \geq 1$. Moreover, it follows that

$$
\lambda t_{0}^{2}(\lambda-\Delta)^{-1}\left(h(x) w^{2}\right) \in \operatorname{Int}\left(P_{e}\right)
$$

since $h(x) w^{2}>0$ in $D$.

Therefore, combining assertions (6.9), (6.10) and (6.11) we obtain that

$$
u-t_{0} r^{*} w \in \operatorname{Int}\left(P_{e}\right) \text {. }
$$

This contradicts condition (6.8).

By Claim 6.1, it follows that the Fréchet derivative $I-H_{v}(\lambda, u)$ is invertible in $C_{e}(\bar{D})$. Hence, by using the implicit function theorem we can find a positive bifurcation solution curve $(\lambda, \tilde{u}(\lambda))$ of the equation $u=H(\lambda, u)$ for all $\lambda \in\left(\lambda_{1}(m), \lambda^{*}\right)$.

The proof of Lemma 6.4 is complete.

\section{Proof of Theorem $1.3-(2)-$}

It remains to characterize explicitly the critical value $\bar{\lambda}(h)$ in Lemma 6.4 as follows:

$$
\bar{\lambda}(h)=\mu_{1}\left(D_{0}(h)\right) .
$$

Step I: First, we consider the logistic Dirichlet problem

$$
\begin{cases}-\Delta v=\lambda(m(x)-h(x) v) v & \text { in } D, \\ v=0 & \text { on } \partial D .\end{cases}
$$

Then we have the following generalization of Cantrell and Cosner [5, Theorems 2.1 and 2.3], Hess [12, Theorem 27.1] and Hess and Kato [13, Theorem 2] to the case where $h(x)$ may vanish in $D$ :

Theorem 7.1. Assume that the function $m(x) \in C^{\theta}(\bar{D})$ satisfies condition (H.1), and further that the function $h(x) \in C^{1}(\bar{D})$ satisfies condition $(\mathrm{Z})$ and that each set $\left\{x \in D_{0}^{i}(h): m(x)>0\right\}, 1 \leq i \leq \ell$, has positive measure. Then the logistic Dirichlet problem (7.2) has a unique positive solution $v(\lambda) \in C^{2+\theta}(\bar{D})$ for every $\lambda \in\left(\gamma_{1}(m), \mu_{1}\left(D_{0}(h)\right)\right)$. For any $\lambda \geq \mu_{1}\left(D_{0}(h)\right)$, there exists no positive solution of problem (7.2). Moreover, we have

$$
\lim _{\lambda \rightarrow \mu_{1}\left(D_{0}(h)\right)}\|v(\lambda)\|_{L^{2}(D)}=+\infty
$$


and also

$$
\lim _{\lambda \rightarrow \gamma_{1}(m)}\|v(\lambda)\|_{C^{2+\theta}(\bar{D})}=0 .
$$

Theorem 7.1 is proved by Taira [25, Theorem 1.2]. The situation may be represented schematically by the following bifurcation diagram, Figure 7.1:

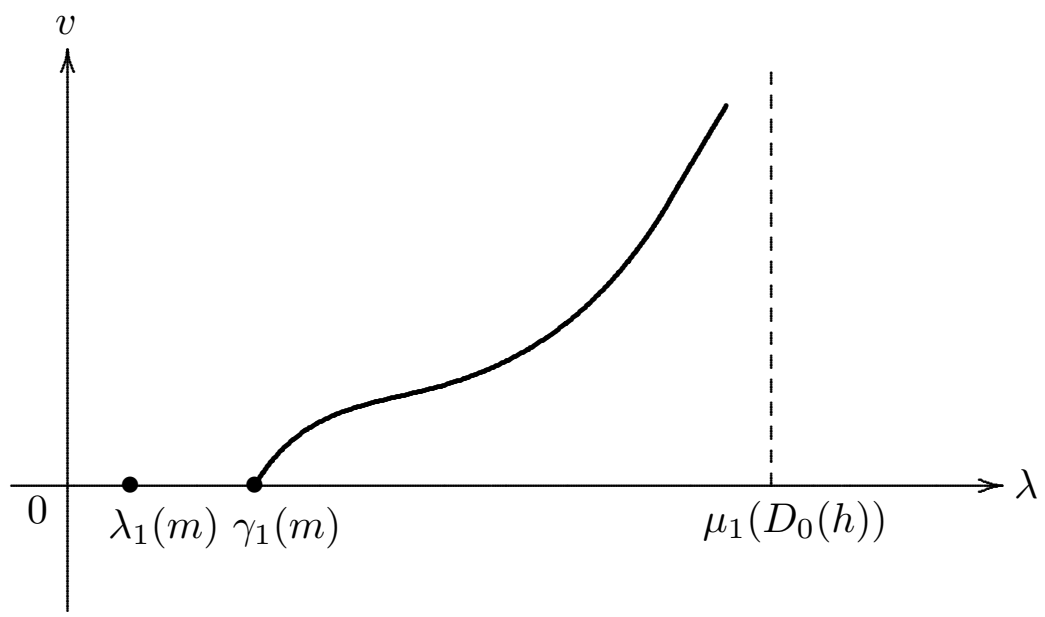

FiguRe 7.1

Remark 7.1. López-Gómez and Sabina de Lis [16] analyze the pointwise growth to infinity of positive solutions of the logistic Dirichlet problem under the condition that $m(x) \equiv 1$ in $D$ (see [16, Theorems 4.2 and 4.3]). Moreover, García-Melián et al [10] study the pointwise behavior and the uniqueness of positive solutions of nonlinear elliptic boundary value problems of general sublinear type, and give the exact limiting profile of the positive solutions (see [10, Theorem 3.1, Corollary 3.3 and Theorem 6.4]). Their numerical computations confirm and illuminate the above bifurcation diagram, Figure 7.1.

Step II: Next we consider the logistic Neumann problem

$$
\begin{cases}-\Delta w=\lambda(m(x)-h(x) w) w & \text { in } D, \\ \frac{\partial w}{\partial \mathbf{n}}=0 & \text { on } \partial D .\end{cases}
$$

Then we have the following generalization of Hess [12, Theorem 27.1] and Senn [21, Theorem 2.4] to the case where $h(x)$ may vanish in $D$ :

Theorem 7.2. Assume that the function $m(x) \in C^{\theta}(\bar{D})$ satisfies condition (H.3), and further that the function $h(x) \in C^{1}(\bar{D})$ satisfies condition $(\mathrm{Z})$ and that each set $\left\{x \in D_{0}^{i}(h): m(x)>0\right\}, 1 \leq i \leq \ell$, has positive measure. Then the logistic Neumann problem (7.3) has a unique positive solution $w(\lambda) \in C^{2+\theta}(\bar{D})$ for every 
$\lambda \in\left(\nu_{1}(m), \mu_{1}\left(D_{0}(h)\right)\right)$. For any $\lambda \geq \mu_{1}\left(D_{0}(h)\right)$, there exists no positive solution of problem (7.3). Moreover, we have

$$
\lim _{\lambda \rightarrow \mu_{1}\left(D_{0}(h)\right)}\|w(\lambda)\|_{L^{2}(D)}=+\infty,
$$

and also

$$
\lim _{\lambda \rightarrow \nu_{1}(m)}\|w(\lambda)-c\|_{C^{2+\theta}(\bar{D})}=0
$$

where

$$
c=\max \left\{\frac{\int_{D} m(x) d x}{\int_{D} h(x) d x}, 0\right\} .
$$

Theorem 7.2 is proved by Taira [25, Theorem 7.2]. The situation may be represented schematically by the three bifurcation diagrams, Figures 7.2, 7.3 and 7.4 in the next page.

Step III: The next comparison principle plays an essential role in the proof of formula (7.1):

Theorem 7.3. Assume that conditions (H.2) and (H.3) are satisfied. If $u(\lambda), v(\lambda)$ and $w(\lambda)$ are two positive solutions of problems (1.1), (7.2) and (7.3), respectively, then we have the inequalities (see Figures 7.5, 7.6 and 7.7)

$$
v(\lambda) \leq u(\lambda) \leq w(\lambda) \quad \text { on } \bar{D} .
$$

Proof. (1) First, we show that

$$
u(\lambda) \leq w(\lambda) \quad \text { on } \bar{D} .
$$

Let

$$
\varphi(x)=u(\lambda)(x)-w(\lambda)(x)
$$

and assume, to the contrary, that the set

$$
D^{+}=\{x \in D: \varphi(x)>0\}=\{x \in D: u(\lambda)(x)>w(\lambda)(x)\}
$$

is non-empty. Then it follows that

$$
\begin{aligned}
0 & =-\Delta \varphi-\lambda\left(m(x) u(\lambda)-h(x) u(\lambda)^{2}\right)+\lambda\left(m(x) w(\lambda)-h(x) w(\lambda)^{2}\right) \\
& =-\Delta \varphi-\lambda m(x) \varphi+\lambda h(x)\left(u(\lambda)^{2}-w(\lambda)^{2}\right) \\
& =-\Delta \varphi-\lambda m(x) \varphi+\lambda h(x)(u(\lambda)+w(\lambda)) \varphi \text { in } D .
\end{aligned}
$$

Hence we have

$$
\Delta \varphi+\lambda m(x) \varphi=\lambda h(x)(u(\lambda)+w(\lambda)) \varphi \geq 0 \text { in } D^{+} .
$$

Let $x_{0}$ be a point of the closure $\overline{D^{+}}$such that

$$
\varphi\left(x_{0}\right)=\frac{\max }{D^{+}} \varphi(x)>0 .
$$

Without loss of generality, we may assume that

$$
\sup _{x \in D} m(x) \leq 1 .
$$




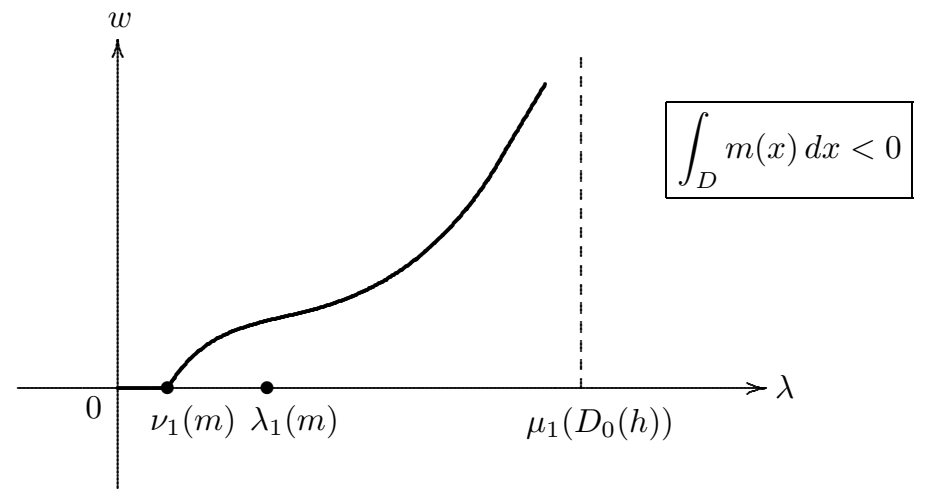

FIGURE 7.2

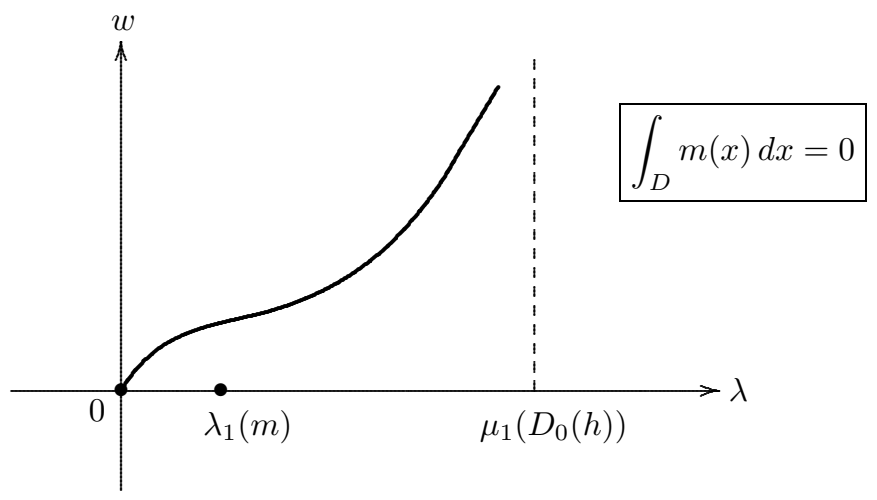

Figure 7.3

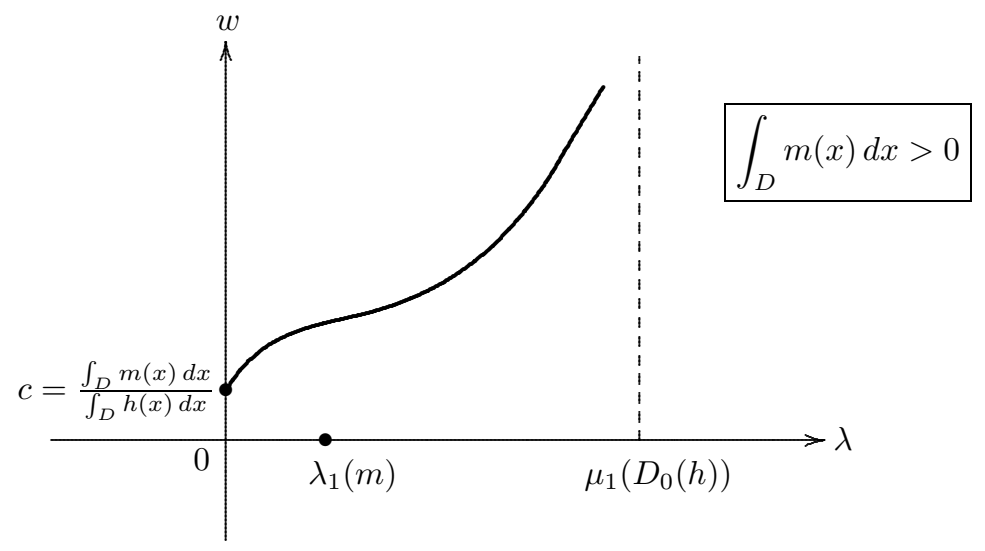

Figure 7.4 
If we let

$$
\Phi(x, t)=e^{-\lambda t} \varphi(x), \quad x \in D, t \geq 0
$$

then it follows from inequality (7.5) that the function $\Phi(x, t)$ satisfies the inequality

$$
\begin{aligned}
\frac{\partial \Phi}{\partial t}-\Delta \Phi+\lambda(1-m(x)) \Phi & =e^{-\lambda t}(-\Delta \varphi-\lambda m(x) \varphi) \\
& \leq 0 \quad \text { in } D^{+} \times(0, T)
\end{aligned}
$$

Here we remark, by condition (7.7), that

$$
\lambda(1-m(x)) \geq 0 \quad \text { in } D
$$

and that

$$
\max _{D^{+} \times[0, T]} \Phi(x, t)=\frac{\max }{D^{+}} \varphi(x)=\varphi\left(x_{0}\right)>0 .
$$

(a) We consider the case where $x_{0} \in D^{+}$: By applying the parabolic maximum principle (see [19, Chapter 3, Section 3, Theorem 7]) to our situation, we obtain from condition (7.6) that

$$
\varphi(x)=\Phi(x, 0) \equiv \Phi\left(x_{0}, 0\right)=\varphi\left(x_{0}\right)>0, \quad x \in D^{+} .
$$

However, this is a contradiction, since we have

$$
\varphi(x)=0 \quad \text { on } \partial D^{+} \cap D .
$$

(b) Next we consider the case where $x_{0}^{\prime} \in \partial D \cap \partial D^{+}$: Then it follows from an application of the Hopf boundary point lemma (Theorem 3.4) that

$$
\frac{\partial \varphi}{\partial \mathbf{n}}\left(x_{0}^{\prime}\right)>0
$$

However, we have

$$
0=B \varphi\left(x_{0}^{\prime}\right)=a\left(x_{0}^{\prime}\right) \frac{\partial \varphi}{\partial \mathbf{n}}\left(x_{0}^{\prime}\right)+b\left(x_{0}^{\prime}\right) \varphi\left(x_{0}^{\prime}\right) .
$$

Thus, combining conditions (7.6) and (7.8) we obtain that

$$
a\left(x_{0}^{\prime}\right)=b\left(x_{0}^{\prime}\right)=0 .
$$

This contradicts condition (H.2).

Therefore, we have proved assertion (7.4), since the set $D^{+}$is empty.

(2) Secondly, we show that

$$
v(\lambda) \leq u(\lambda) \text { on } \bar{D} .
$$

Let

$$
\psi(x)=v(\lambda)(x)-u(\lambda)(x),
$$

and assume, to the contrary, that the set

$$
E^{+}=\{x \in D: \psi(x)>0\}=\{x \in D: u(\lambda)(x)>w(\lambda)(x)\}
$$


is non-empty. Then it follows that

$$
\begin{aligned}
0 & =-\Delta \psi-\lambda\left(m(x) v(\lambda)-h(x) v(\lambda)^{2}\right)+\lambda\left(m(x) u(\lambda)-h(x) u(\lambda)^{2}\right) \\
& =-\Delta \psi-\lambda m(x) \psi+\lambda h(x)\left(v(\lambda)^{2}-u(\lambda)^{2}\right) \\
& =-\Delta \psi-\lambda m(x) \psi+\lambda h(x)(v(\lambda)+u(\lambda)) \psi \text { in } D .
\end{aligned}
$$

Hence we have

$$
\Delta \psi+\lambda m(x) \psi=\lambda h(x)(v(\lambda)+u(\lambda)) \psi \geq 0 \quad \text { in } E^{+} .
$$

Let $x_{0}$ be a point of the closure $\overline{E^{+}}$such that

$$
\psi\left(x_{0}\right)=\max _{\overline{E^{+}}} \psi(x)>0 .
$$

If we let

$$
\Psi(x, t)=e^{-\lambda t} \psi(x), \quad x \in D, t \geq 0,
$$

then it follows from inequality (7.10) that the function $\Psi(x, t)$ satisfies the inequality

$$
\begin{aligned}
\frac{\partial \Psi}{\partial t}-\Delta \Psi+\lambda(1-m(x)) \Psi & =e^{-\lambda t}(-\Delta \psi-\lambda m(x) \psi) \\
& \leq 0 \text { in } D^{+} \times(0, T) .
\end{aligned}
$$

Here we recall, by condition (7.7), that

$$
\lambda(1-m(x)) \geq 0 \quad \text { in } D,
$$

and that

$$
\max _{E^{+} \times[0, T]} \Psi(x, t)=\max _{\bar{E}^{+}} \psi(x)=\psi\left(x_{0}\right)>0 .
$$

(a) We consider the case where $x_{0} \in E^{+}$: By applying the parabolic maximum principle (see [19, Chapter 3, Section 3, Theorem 7]) to our situation, we obtain that

$$
\psi(x)=\Psi(x, 0) \equiv \Psi\left(x_{0}, 0\right)=\psi\left(x_{0}\right)>0, \quad x \in E^{+} .
$$

However, this is a contradiction, since we have

$$
\psi(x)=0 \quad \text { on } \partial E^{+} \cap D .
$$

(b) Next we consider the case where $x_{0}^{\prime} \in \partial D \cap \partial E^{+}$: Then we have, by condition (7.11),

$$
0<\psi\left(x_{0}^{\prime}\right)=v(\lambda)\left(x_{0}^{\prime}\right)-u(\lambda)\left(x_{0}^{\prime}\right)=-u(\lambda)\left(x_{0}^{\prime}\right) \leq 0 .
$$

This is a contradiction.

Therefore, we have proved assertion (7.9), since that the set $E^{+}$is empty.

Now the proof of Theorem 7.3 is complete.

Step IV: The desired formula (7.1) follows by combining Theorem 5.3, Lemmas 6.1 and 6.3, and Theorems 7.1, 7.2 and 7.3. Indeed, it suffices to note the three bifurcation diagrams, Figures 7.5, 7.6 and 7.7 in the next page.

Now the proof of Theorem 1.3 is complete. 


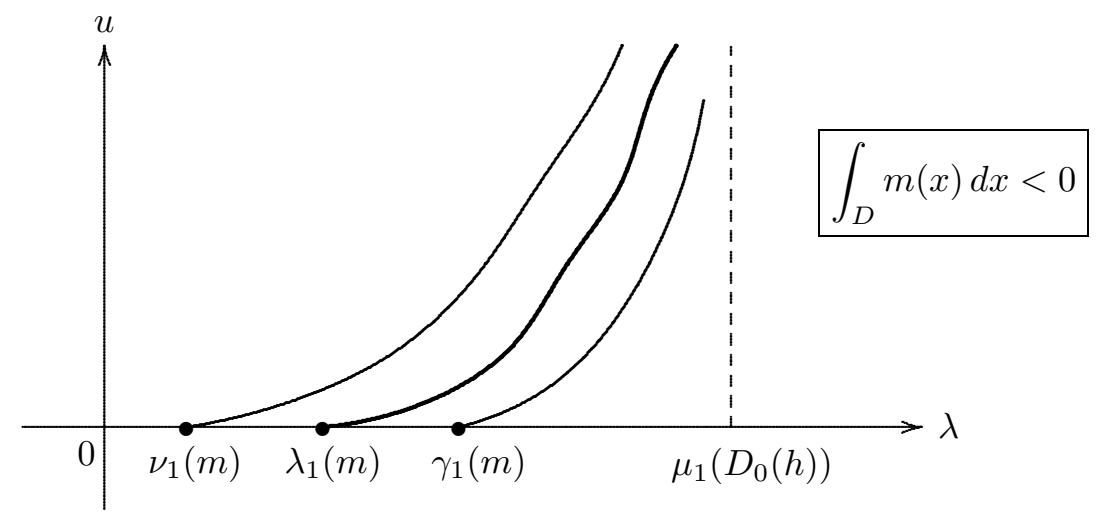

FIGURE 7.5

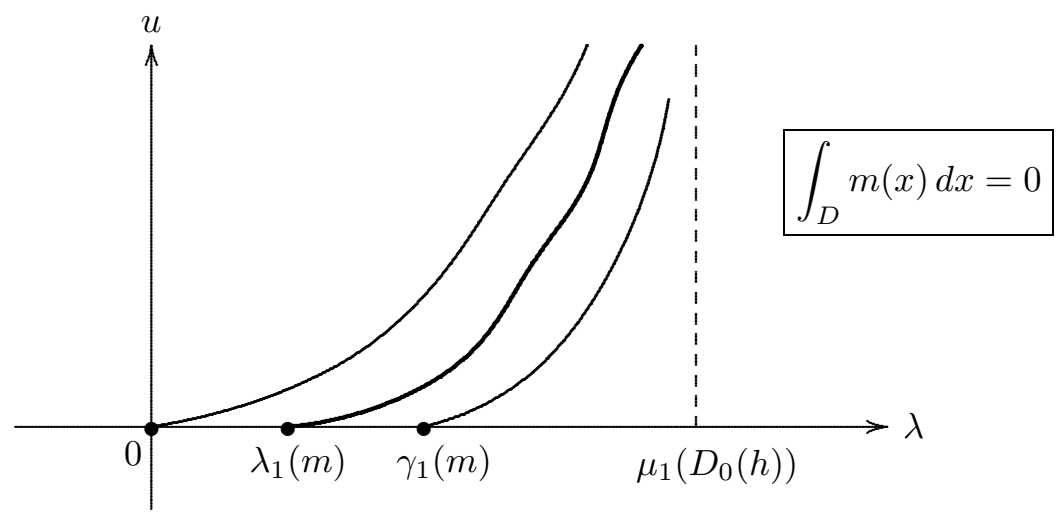

Figure 7.6

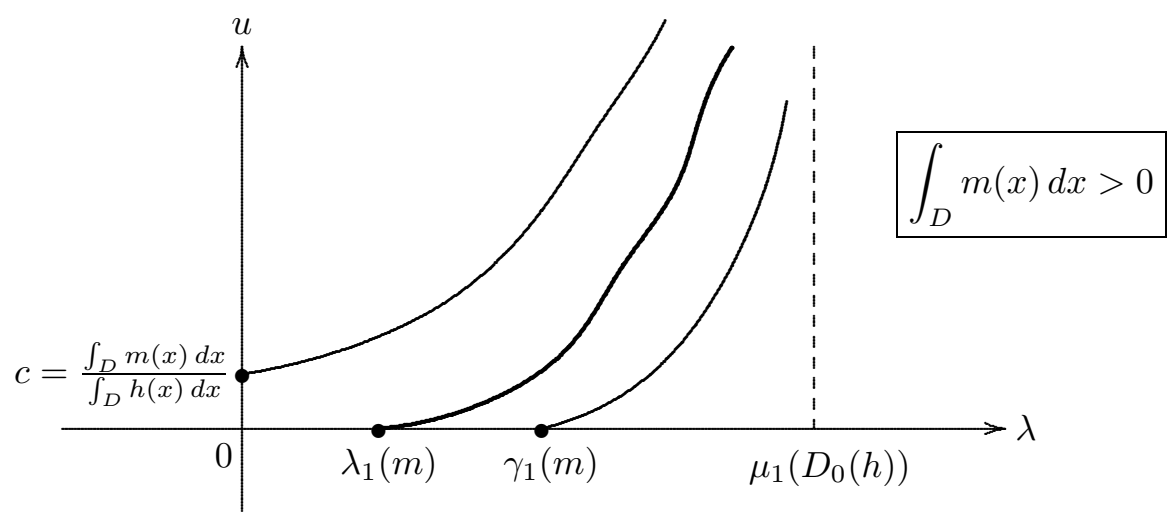

FIGURE 7.7 


\section{Concluding Remarks}

In this section we consider problem (1.1) under the condition that

(P) $h(x)>0$ on $\bar{D}$.

The next theorem is a generalization of Cantrell and Cosner [5, Theorem 2.1] to the degenerate case:

Theorem 8.1. In addition to conditions (H.1) and (H.2), assume that the function $h(x) \in C^{\theta}(\bar{D}), 0<\theta<1$, satisfies condition $(\mathrm{P})$. Then problem (1.1) has a unique positive solution $u(\lambda) \in C^{2+\theta}(\bar{D})$ for every $\lambda>\lambda_{1}(m)$. Moreover, we can give an estimate of the growth rate of the total size $\|u(\lambda)\|_{L^{1}(D)}$ of the positive steady states $u(\lambda)$

$$
\int_{D} u(\lambda) d x \leq\left(1-\frac{\lambda_{1}(m)}{\lambda}\right)|D|^{2 / 3} \frac{\left(\int_{D}\left(m^{+}\right)^{3} d x\right)^{1 / 3}}{\min _{x \in \bar{D}} h(x)}, \quad \lambda>\lambda_{1}(m) .
$$

Here $|D|$ is the volume of the domain $D$ and $m^{+}(x)=\max \{m(x), 0\}, x \in D$.

Remark 8.1. It should be emphasized that $\bar{\lambda}(h)=+\infty$ if the function $h(x)$ satisfies condition $(\mathrm{P})$. More precisely, by using the maximum principle as in Cantrell and Cosner [5] we can give a uniform bound on the positive steady states $u(\lambda)$

$$
\max _{\bar{D}} u(\lambda) \leq \ell:=\frac{\max _{\bar{D}} m^{+}}{\min _{\bar{D}} h}, \quad \lambda>\lambda_{1}(m) .
$$

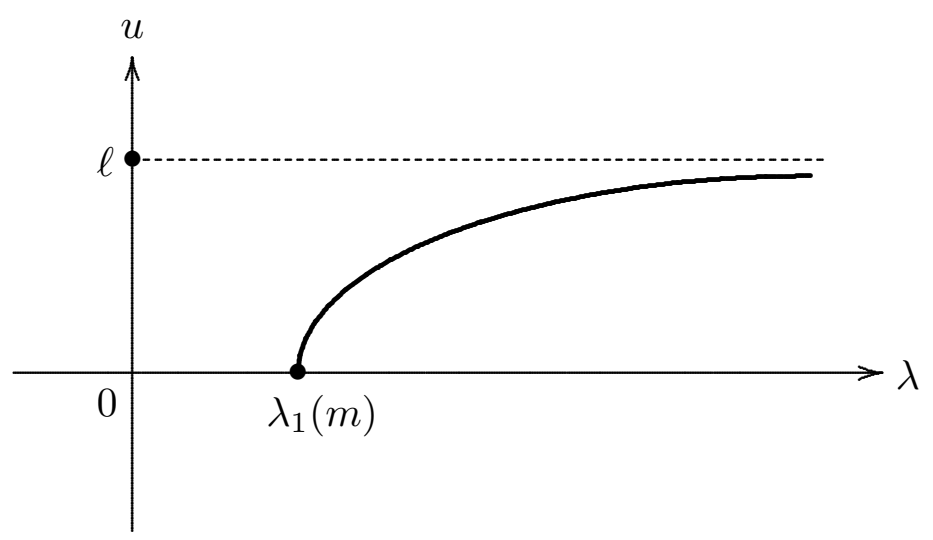

FiguRE 8.1

Rephrased, Theorem 8.1 asserts that the models we consider predict persistence for a population if its diffusion rate $1 / \lambda$ is below the critical value $1 / \lambda_{1}(m)$, and predict extinction for a population if the diffusion rate $1 / \lambda$ is above the critical value $1 / \lambda_{1}(m)$. The size of $\lambda_{1}(m)$ is of crucial importance; increasing $\lambda_{1}(m)$ imposes a more stringent condition on the diffusion rate $1 / \lambda$ if the population is to persist, since $0<1 / \lambda<1 / \lambda_{1}(m)$. 
The situation may be represented schematically by the above bifurcation diagram, Figure 8.1:

\subsection{Proof of Theorem 8.1}

In view of Lemmas 6.1 and 6.3 , it suffices to prove estimate (8.1) of the total size of the positive steady states $u(\lambda)$.

First, we have, by Green's formula,

$$
\begin{aligned}
& \lambda\left(\int_{D} m(x) u(\lambda)^{2} d x-\int_{D} h(x) u(\lambda)^{3} d x\right) \\
= & -\int_{D} \Delta u(\lambda) \cdot u(\lambda) d x \\
= & \int_{D}|\nabla u(\lambda)|^{2} d x-\int_{\partial D} \frac{\partial u(\lambda)}{\partial \mathbf{n}} u(\lambda) d \sigma \\
= & \int_{D}|\nabla u(\lambda)|^{2} d x+\int_{\left\{a\left(x^{\prime}\right) \neq 0\right\}} \frac{b\left(x^{\prime}\right)}{a\left(x^{\prime}\right)} \cdot u(\lambda)^{2} d \sigma,
\end{aligned}
$$

and so

$$
\begin{aligned}
0 & <\frac{1}{\lambda}\left(\int_{D}|\nabla u(\lambda)|^{2} d x+\int_{\left\{a\left(x^{\prime}\right) \neq 0\right\}} \frac{b\left(x^{\prime}\right)}{a\left(x^{\prime}\right)} \cdot u(\lambda)^{2} d \sigma\right)+\int_{D} h(x) u(\lambda)^{3} d x \\
& =\int_{D} m(x) u(\lambda)^{2} d x
\end{aligned}
$$

By applying the variational formula (1.5), we obtain that

$$
\begin{aligned}
(-\Delta u(\lambda), u(\lambda))_{L^{2}(D)} & =\int_{D}|\nabla u(\lambda)|^{2} d x+\int_{\left\{a\left(x^{\prime}\right) \neq 0\right\}} \frac{b\left(x^{\prime}\right)}{a\left(x^{\prime}\right)} \cdot u(\lambda)^{2} d \sigma \\
& \geq \lambda_{1}(m) \int_{D} m(x) u(\lambda)^{2} d x
\end{aligned}
$$

Hence it follows from formula (8.3) and inequality (8.4) that

$$
\begin{aligned}
& \int_{D} h(x) u(\lambda)^{3} d x \\
= & \int_{D} m(x) u(\lambda)^{2} d x-\frac{1}{\lambda}\left(\int_{D}|\nabla u(\lambda)|^{2} d x+\int_{\left\{a\left(x^{\prime}\right) \neq 0\right\}} \frac{b\left(x^{\prime}\right)}{a\left(x^{\prime}\right)} u(\lambda)^{2} d \sigma\right) \\
\leq & \int_{D} m(x) u(\lambda)^{2} d x-\frac{\lambda_{1}(m)}{\lambda} \int_{D} m(x) u(\lambda)^{2} d x \\
= & \left(1-\frac{\lambda_{1}(m)}{\lambda}\right) \int_{D} m(x) u(\lambda)^{2} d x .
\end{aligned}
$$


Secondly, we have, by Hölder's inequality,

$$
\begin{aligned}
\int_{D} m(x) u(\lambda)^{2} d x & \leq \int_{D} m^{+}(x) u(\lambda)^{2} d x \\
& \leq\left(\int_{D}\left(m^{+}(x)\right)^{3} d x\right)^{1 / 3}\left(\int_{D} u(\lambda)^{3} d x\right)^{2 / 3} \\
& =\left\|m^{+}\right\|_{L^{3}(D)}\left(\|u(\lambda)\|_{L^{3}(D)}\right)^{2}
\end{aligned}
$$

By combining inequalities (8.5) and (8.6), we obtain that

$$
\begin{aligned}
\min _{x \in \bar{D}} h(x)\left(\|u(\lambda)\|_{L^{3}(D)}\right)^{3} & \leq \int_{D} h(x) u(\lambda)^{3} d x \\
& \leq\left(1-\frac{\lambda_{1}(m)}{\lambda}\right) \int_{D} m(x) u(\lambda)^{2} d x \\
& \leq\left(1-\frac{\lambda_{1}(m)}{\lambda}\right)\left\|m^{+}\right\|_{L^{3}(D)}\left(\|u(\lambda)\|_{L^{3}(D)}\right)^{2}
\end{aligned}
$$

This proves that

$$
\|u(\lambda)\|_{L^{3}(D)} \leq\left(1-\frac{\lambda_{1}(m)}{\lambda}\right) \frac{\left\|m^{+}\right\|_{L^{3}(D)}}{\min _{x \in \bar{D}} h(x)} .
$$

On the other hand, by Hölder's inequality it follows that

$$
\int_{D} u(\lambda) d x \leq\left(\int_{D} u(\lambda)^{3} d x\right)^{1 / 3}\left(\int_{D} d x\right)^{2 / 3}=|D|^{2 / 3}\|u(\lambda)\|_{L^{3}(D)} .
$$

Therefore, the desired estimate (8.1) follows by combining inequalities (8.7) and (8.8).

Now the proof of Theorem 8.1 is complete.

\subsection{Proof of Remark 8.1}

Now we prove the uniform estimate (8.2).

If we let

$$
w(x) \equiv \ell:=\frac{\max _{\bar{D}} m^{+}}{\min _{\bar{D}} h}
$$

then we have

$$
\begin{aligned}
-\Delta w-\lambda m(x) w+\lambda h(x) w^{2} & =-\lambda m(x) \ell+\lambda h(x) \ell^{2} \\
& \geq \lambda \ell(h(x) \ell-m(x)) \\
& \geq 0 \text { in } D,
\end{aligned}
$$

and

$$
B w=b\left(x^{\prime}\right) \ell \geq 0 \quad \text { on } \partial D .
$$


This proves that the function $w(x)$ is a supersolution of problem (1.1). Therefore, using a comparison theorem based on the maximum principle we obtain the uniform estimate (8.2)

$$
0 \leq u(\lambda) \leq w \equiv \ell \quad \text { in } D
$$

\section{References}

[1] H. Amann, Fixed point equations and nonlinear eigenvalue problems in ordered Banach spaces. SIAM Rev. 18 (1976), 620-709.

[2] J. Bergh and J. Löfström, Interpolation spaces, an introduction. Springer-Verlag, Berlin Heidelberg New York, 1976.

[3] J.-M. Bony, Principe du maximum dans les espaces de Sobolev. C. R. Acad. Sc. Paris 265 (1967), 333-336.

[4] K.J. Brown and S.S. Lin, On the existence of positive eigenfunctions for an eigenvalue problem with indefinite weight function. J. Math. Anal. Appl. 75 (1980), 112-120.

[5] R.S. Cantrell and C. Cosner, Diffusive logistic equations with indefinite weights: population models in disrupted environments. Proc. Roy. Soc. Edinburgh 112A (1989), 293-318.

[6] M.G. Crandall and P.H. Rabinowitz, Bifurcation from simple eigenvalues. J. Functional Analysis 8 (1971), 321-340.

[7] D.G. de Figueiredo, Positive solutions of semilinear elliptic problems, Lecture Notes in Mathematics, No. 957, Springer-Verlag, Berlin Heidelberg New York, (1982), $34-87$.

[8] K. Deimling, Nonlinear functional analysis, Springer-Verlag, Berlin Heidelberg New York Tokyo, 1985.

[9] J.M. Fraile, P. Koch Medina, J. López-Gómez and S. Merino, Elliptic eigenvalue problems and unbounded continua of positive solutions of a semilinear elliptic equation. J. Differential Equations 127 (1996), 295-319.

[10] J. García-Melián, R. Gómez-Reñasco, J. López-Gómez and J.C. Sabina de Lis, Pointwise growth and uniqueness of positive solutions for a class of sublinear elliptic problems where bifurcation from infinity occurs. Arch. Rational Mech. Anal. 145 (1998), 261-289.

[11] D. Gilbarg and N.S. Trudinger, Elliptic partial differential equations of second order, 1998 edition, Springer-Verlag, New York Berlin Heidelberg Tokyo, 1998.

[12] P. Hess, Periodic-parabolic boundary value problems and positivity, Pitman Research Notes in Mathematical Series 247, Longman Scientific \& Technical, Harlow, Essex, 1991.

[13] P. Hess and T. Kato, On some linear and nonlinear eigenvalue problems with an indefinite weight function. Comm. Partial Differential Equations 5 (1980), 999-1030.

[14] M.A. Krasnosel'skii, Positive solutions of operator equations, P. Noordhoff, Groningen, 1964.

[15] M.G. KreĬn and M.A. Rutman, Linear operators leaving invariant a cone in a Banach space. Amer. Math. Soc. Transl. 10 (1962) 199-325. 
[16] J. López-Gómez and J.C. Sabina de Lis, First variations of principal eigenvalues with respect to the domain and point-wise growth of positive solutions for problems where bifurcation from infinity occurs. J. Differential Equations 148 (1998), 47-64.

[17] A. Manes and A.M. Micheletti, Un'estensione della teoria variazionale classica degli autovalori per operatori ellitici del secondo ordine. Boll. Un. Mat. Ital. 7 (1973), 285-301.

[18] T.C. Ouyang, On the positive solutions of semilinear equations $\Delta u+\lambda u-h u^{p}=0$ on the compact manifolds. Trans. Amer. Math. Soc. 331 (1992), 503-527.

[19] M.H. Protter and H.F. Weinberger, Maximum principles in differential equations, Prentice-Hall, Englewood Cliffs, New Jersey, 1967.

[20] P.H. Rabinowitz, Some aspects of nonlinear eigenvalue problems. Rocky Mountain J. Math. 3 (1973), 161-202.

[21] S. Senn, On a nonlinear elliptic eigenvalue problem with Neumann boundary conditions, with an application to population genetics. Comm. Partial Differential Equations 8 (1983), 1199-1228.

[22] S. Senn and P. Hess, On positive solutions of a linear elliptic eigenvalue problem with Neumann boundary conditions. Math. Ann. 258 (1982), 459-470.

[23] K. Taira, Introduction to semilinear elliptic boundary value problems. Taiwanese J. Math. 2 (1998), 127-172.

[24] K. Taira, Positive solutions of diffusive logistic equations, Taiwanese J. Math. 5 (2001), 117-140.

[25] K. Taira, Introduction to diffusive logistic equations in population dynamics, Korean J. Comput. Appl. Math. 9 (2002), 289-347.

[26] K. Taira, Logistic Dirichlet problems with discontinuous coefficients. J. Math. Pures Appl. 82 (2003), 1137-1190.

[27] K. Taira, Semigroups, boundary value problems and Markov processes, SpringerVerlag, Berlin Heidelberg New York, 2004.

[28] H. Triebel, Theory of function spaces, Birkhäuser, Basel Boston Stuttgart, 1983.

[29] G.M. Troianiello, Elliptic differential equations and obstacle problems, Plenum Press, New York, 1987.

[30] K. Yosida, Functional analysis, sixth edition, Springer-Verlag, Berlin Heidelberg New York, 1980.

Kazuaki Taira

Institute of Mathematics

University of Tsukuba

Tsukuba 305-8571

Japan

e-mail: taira@math.tsukuba.ac.jp 